\title{
Aryldiazonium Salts Serve as Dual Synthon: \\ Construction of Fully Substituted Pyrazoles via Rongalite-Mediated Three-Component Radical Annulation Reaction
}

Miao Wang, ${ }^{\mathrm{a}, \mathrm{b}}$ Bo-Cheng Tang, ${ }^{\mathrm{b}}$ Jia-Chen Xiang, ${ }^{\mathrm{b}}$ Xiang-Long Chen, ${ }^{\mathrm{b}}$ Jin-Tian Ma, ${ }^{\mathrm{b}}$ Yan-Dong $\mathrm{Wu}$, ${ }^{\mathrm{b}}$ and $\mathrm{An}-\mathrm{Xin} \mathrm{Wu}{ }^{*} \mathrm{~b}$

${ }^{\text {a }}$ College of Chemistry and Chemical Engineering, Henan Key Laboratory of Function-Oriented Porous Materials, Luoyang Normal University, Luoyang 471022, P. R. China

${ }^{\mathrm{b}}$ Key Laboratory of Pesticide \& Chemical Biology, Ministry of Education, College of Chemistry, Central China Normal University, Hubei, Wuhan 430079, P. R. China

E-mail: chwuax@mail.ccnu.edu.cn

Table of Contents

page

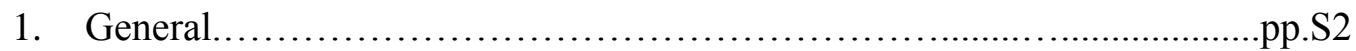

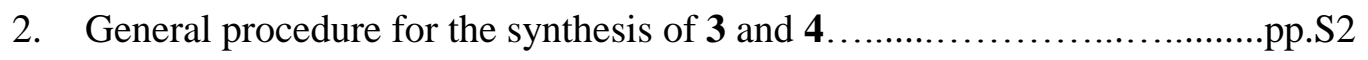

3. Evidence in support of the mechanism.......................................pp.S3

4. Crystallographic data of 3 e..................................................Sp 4 .S

5. Characterization data for target compound.......................................Sp.S6-S15

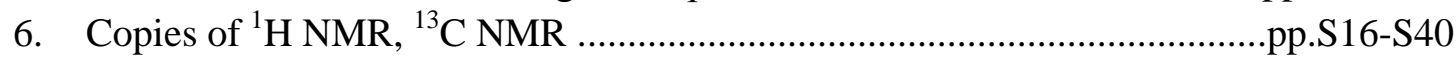




\section{General.}

All kinds of aryldiazonium tetrafluoroborates 1a-1p were prepared according to literature procedures ${ }^{1}$ and other substrates and reagents were commercially available and used without further purification. TLC analysis was performed using pre-coated glass plates. Column chromatography was performed using silica gel (200-300 mesh). IR spectra were recorded on a Perkin-Elmer PE-983 infrared spectrometer as $\mathrm{KBr}$ pellets with absorption in $\mathrm{cm}^{-1} .{ }^{1} \mathrm{H}$ spectra were recorded in $\mathrm{CDCl} 3$ or DMSO- $d_{6}$ on $400 / 600 \mathrm{MHz}$ NMR spectrometers and resonances $(\delta)$ are given in parts per million relative to tetramethylsilane. Data are reported as follows: chemical shift, multiplicity ( $\mathrm{s}=$ singlet, $\mathrm{d}=$ doublet, $\mathrm{t}=$ triplet, $\mathrm{m}=$ multiplet, $\mathrm{q}=$ quadruple), coupling constants $(\mathrm{Hz})$ and integration. ${ }^{13} \mathrm{C}$ spectra were recorded in $\mathrm{CDCl}_{3}$ or DMSO-d6 on $100 / 150$ MHz NMR spectrometers and resonances $(\delta)$ are given in ppm. HRMS were obtained on a Bruker 7-tesla FT-ICR MS equipped with an electrospray source. The X-ray crystal structure determinations of $\mathbf{3 e}$ were obtained on a Bruker SMART APEX CCD system. Melting points were determined using XT-4 apparatus and not corrected.

\section{General procedure for the synthesis of 3 and 4 (3a as an example).}

A mixture of benzenediazonium tetrafluoroborate $1 \mathrm{a} \quad(1.0 \mathrm{mmol})$, benzylideneacetone $(2.0 \mathrm{mmol})$ and rongalite $(2.0 \mathrm{mmol})$ in DMSO $(3.0 \mathrm{~mL})$ was stirred at room temperature for $30 \mathrm{~min}$ in a pressure vessel. The resulting mixture was dropped into $50 \mathrm{~mL} \mathrm{H}_{2} \mathrm{O}$ and extracted with EtOAc 3 times $(3 \times 50 \mathrm{~mL})$. The organic extract was dried with anhydrous $\mathrm{Na}_{2} \mathrm{SO}_{4}$, filtered and concentrated. The crude product was purified by column chromatography on silica gel (eluent: petroleum ether $/$ EtOAc $=10 / 1)$ to afford the product 3a as yellow solid $(109.6 \mathrm{mg}, 71 \%)$. 


\section{Evidence in support of the mechanism.}

(1) We performed a series of radical trapping experiments, and such as TEMPO, BHT, and hydroquinone, no desired product (4e) was observed for the reaction of TEMPO and BHT, only $16 \%$ yield of the desired product (4e) was observed for the reaction of hydroquinone. And in the reaction containing TEMPO, a TEMPO-PhCO ${ }_{2}$ Et product 5 was detected by GC-MS.
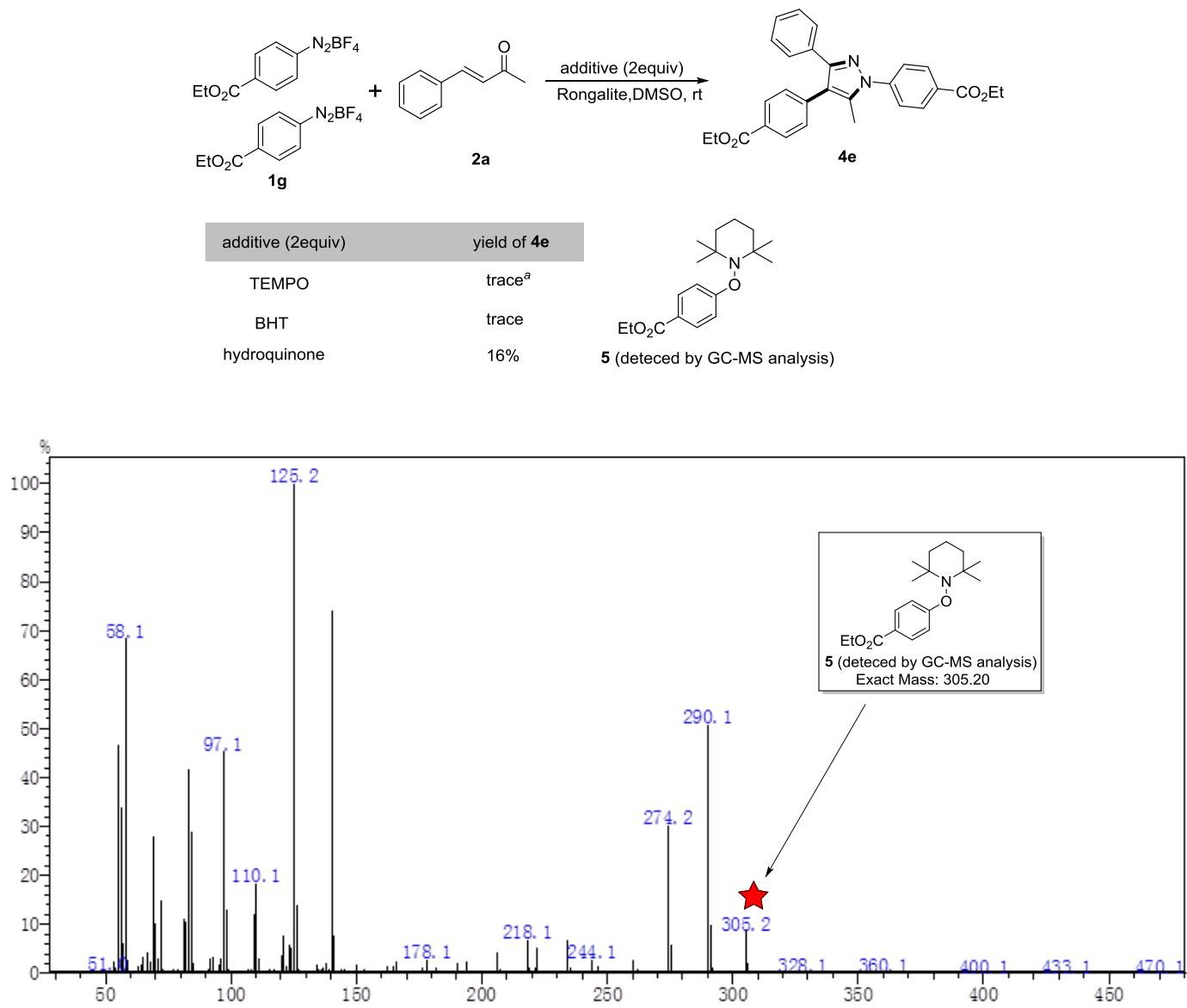

\section{References:}

(1) Wu, J.; Gu,Y.; Leng, X.; Shen, Q. Angew. Chem., Int. Ed., 2015, 54, 7648.

(2) Shu, W. M.; Ma, J. R.; Zheng, K. L.; Wu, A. X. Org. Lett. 2015, 18, 196. 
4. Crystallographic data of $3 e$.<smiles>N#Cc1ccc(-c2cn(-c3ccc(C#N)cc3)nc2-c2ccccc2)cc1</smiles>

4,4'-(3-phenyl-1H-pyrazole-1,4-diyl)dibenzonitrile: (3e)

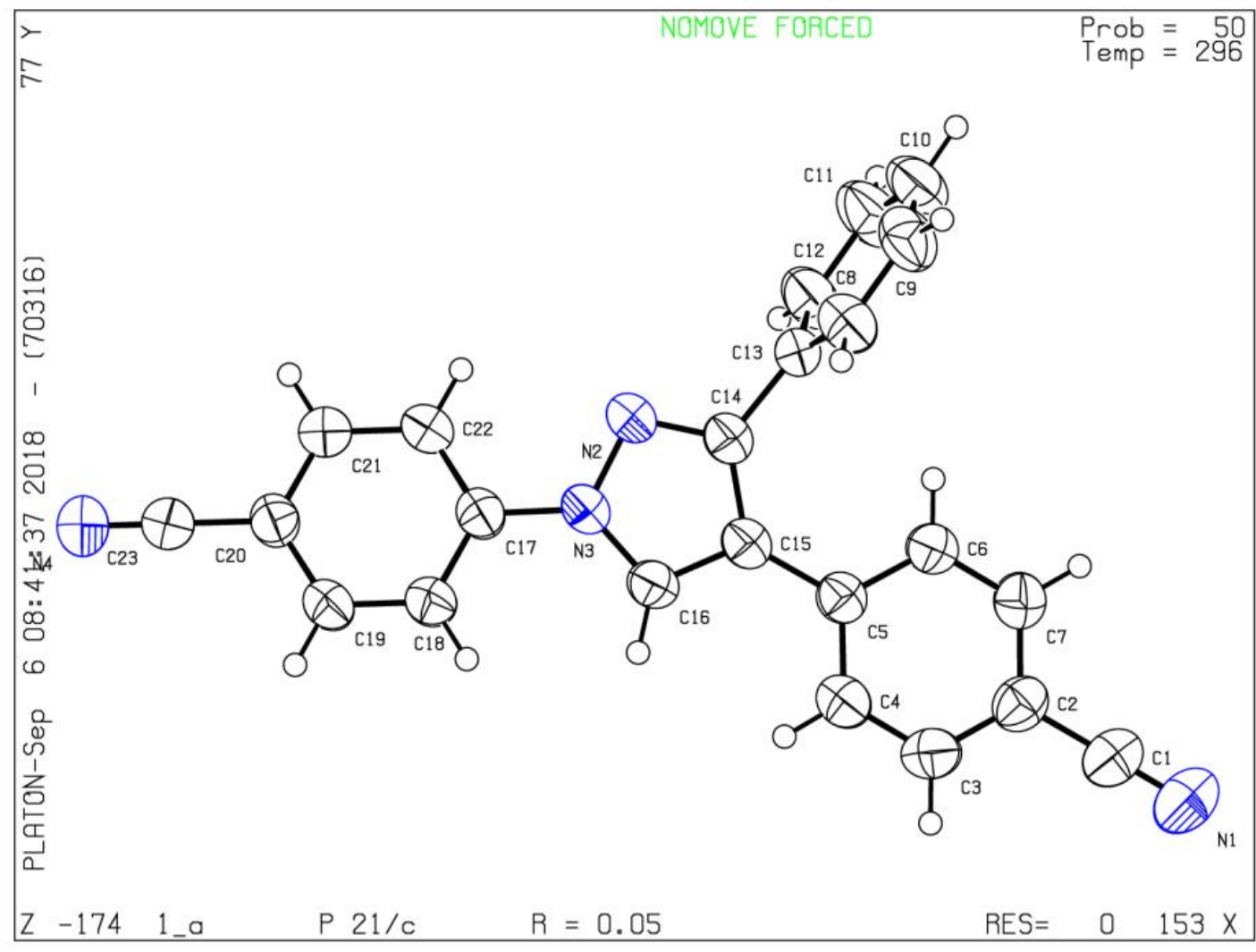

Figure S1. X-ray crystal structure of $3 \mathrm{e}$. 
Table S1. Crystal data and structure refinement for compound 3e (CCDC: 1888713)

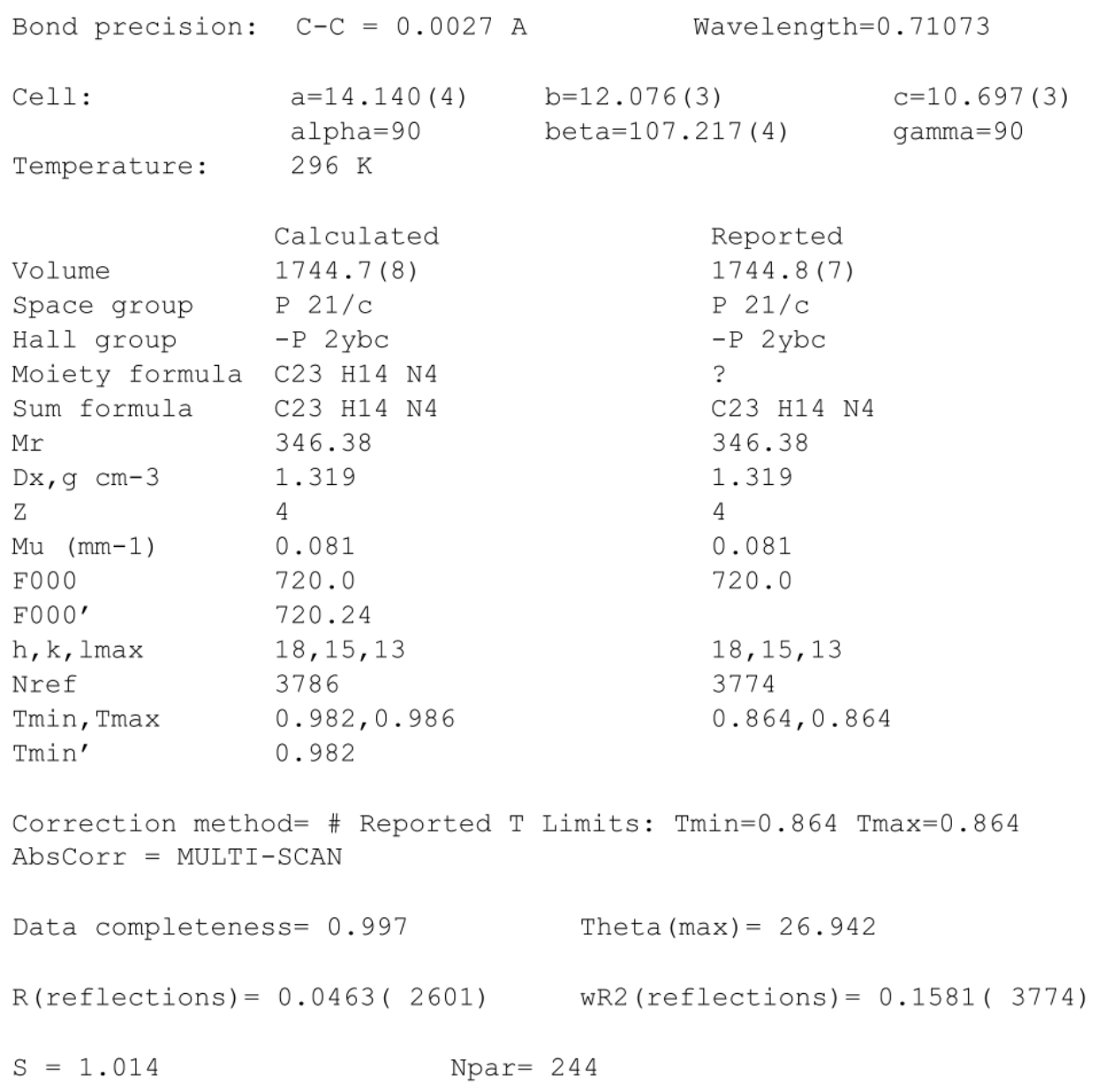




\section{Characterization data for target compound.}<smiles>Cc1c(-c2ccccc2)c(-c2ccccc2)nn1-c1ccccc1</smiles>

3a

\section{5-methyl-1,3,4-triphenyl-1H-pyrazole (3a):}

Yield: 71\% (109.6 mg); yellow solid; IR (KBr): 3441, 2923, 2361, 2335, 1646, 1558, 1453, 1279, $689 \mathrm{~cm}^{-1} ;{ }^{1} \mathrm{H}$ NMR $\left(400 \mathrm{MHz}, \mathrm{CDCl}_{3}\right) \delta=7.59$ (d, J=7.6 Hz, 2H), 7.51$7.49(\mathrm{~m}, 4 \mathrm{H}), 7.42-7.36(\mathrm{~m}, 3 \mathrm{H}), 7.31-7.25(\mathrm{~m}, 6 \mathrm{H}), 2.32(\mathrm{~s}, 3 \mathrm{H}) ;{ }^{13} \mathrm{C}$ NMR $(100 \mathrm{MHz}$, $\left.\mathrm{CDCl}_{3}\right) \delta=149.6,139.9,137.6,133.8,133.2,130.3,129.2,129.1,128.4,128.1,127.7$, 127.4, 126.7, 125.1, 120.0, 11.7 ; HRMS (ESI) m/z calcd for $\mathrm{C}_{22} \mathrm{H}_{19} \mathrm{~N}_{2}{ }^{+}(\mathrm{M}+\mathrm{H})^{+}$ 311.1543 , found 311.1547 .<smiles>Cc1ccc(-c2cn(-c3ccc(C)cc3)nc2-c2ccccc2)cc1</smiles>

\section{3-phenyl-1,4-di-p-tolyl-1H-pyrazole (3b):}

Yield: 62\% (100.1 mg); light yellow oil; IR (KBr): 3442, 2922, 2361, 2336, 1649, 1517, 1450, 1396, 1265, $810 \mathrm{~cm}^{-1} ;{ }^{1} \mathrm{H}$ NMR (600 MHz, $\left.\mathrm{CDCl}_{3}\right) \delta=7.95$ (s, 1H), 7.66 $(\mathrm{d}, J=7.8 \mathrm{~Hz}, 2 \mathrm{H}), 7.60(\mathrm{~d}, J=7.8 \mathrm{~Hz}, 2 \mathrm{H}), 7.33(\mathrm{~d}, J=7.2 \mathrm{~Hz}, 3 \mathrm{H}), 7.261-7.23(\mathrm{~m}$, $4 \mathrm{H}), 7.14(\mathrm{~d}, J=7.2 \mathrm{~Hz}, 2 \mathrm{H}), 2.39(\mathrm{~s}, 3 \mathrm{H}), 2.37(\mathrm{~s}, 3 \mathrm{H}) ;{ }^{13} \mathrm{C} \mathrm{NMR}\left(150 \mathrm{MHz}, \mathrm{CDCl}_{3}\right)$ $\delta=150.1,137.7,136.5,136.2,133.3,129.9,129.2,128.5,128.4,128.2,127.7,126.5$, 125.8, 122.6, 118.9, 21.2, 20.9 ; HRMS (ESI) m/z calcd for $\mathrm{C}_{23} \mathrm{H}_{21} \mathrm{~N}_{2}{ }^{+}(\mathrm{M}+\mathrm{H})^{+}$ 325.1699 , found 325.1694 .<smiles>COc1ccc(-c2c(-c3ccccc3)nn(-c3ccc(OC)cc3)c2C)cc1</smiles>

\section{1,4-bis(4-methoxyphenyl)-5-methyl-3-phenyl-1H-pyrazole (3c):}

Yield: 68\% (125.6 mg); gray solid; IR (KBr): 3443, 2361, 2336, 1648, 1510, 1453, 1370, 1245, 1174, 1036, $832 \mathrm{~cm}^{-1} ;{ }^{1} \mathrm{H}$ NMR $\left(400 \mathrm{MHz}, \mathrm{CDCl}_{3}\right) \delta=7.52(\mathrm{~d}, J=6.4$ $\mathrm{Hz}, 2 \mathrm{H}), 7.48(\mathrm{~d}, J=8.8 \mathrm{~Hz}, 2 \mathrm{H}), 7.40(\mathrm{~s}, 1 \mathrm{H}), 7.24(\mathrm{~s}, 2 \mathrm{H}), 7.19(\mathrm{~d}, J=8.0 \mathrm{~Hz}, 2 \mathrm{H})$, $7.01(\mathrm{~d}, J=8.4 \mathrm{~Hz}, 2 \mathrm{H}), 6.92(\mathrm{~d}, J=8.0 \mathrm{~Hz}, 2 \mathrm{H}), 3.86(\mathrm{~s}, 3 \mathrm{H}), 3.84(\mathrm{~s}, 3 \mathrm{H}), 2.24(\mathrm{~s}$, 
$3 \mathrm{H}) ;{ }^{13} \mathrm{C}$ NMR $\left(150 \mathrm{MHz}, \mathrm{CDCl}_{3}\right) \delta=159.0,158.4,149.1,137.7,133.4,133.0,131.3$, 128.2, 128.1, 128.0, 127.3, 126.6, 126.2, 119.1, 114.2, 113.9, 55.5, 55.2, 11.4; HRMS (ESI) $\mathrm{m} / \mathrm{z}$ calcd for $\mathrm{C}_{24} \mathrm{H}_{23} \mathrm{~N}_{2} \mathrm{O}_{2}{ }^{+}(\mathrm{M}+\mathrm{H})^{+} 371.1754$, found 371.1757 .<smiles>Cc1c(-c2ccccc2)c(-c2ccccc2)nn1-c1cccc(C#N)c1</smiles>

\section{3,3'-(5-methyl-3-phenyl-1H-pyrazole-1,4-diyl)dibenzonitrile (3d):}

Yield: 81\% (145.5 mg); yellow solid; IR (KBr): 3441, 2924, 2361, 2336, 2222, 1648, 1485, 1452, 1368, 1274, $887 \mathrm{~cm}^{-1} ;{ }^{1} \mathrm{H}$ NMR $\left(600 \mathrm{MHz}, \mathrm{CDCl}_{3}\right) \delta=7.95$ (s, 1H), 7.87 $(\mathrm{d}, J=7.8 \mathrm{~Hz}, 1 \mathrm{H}), 7.71(\mathrm{~d}, J=7.8 \mathrm{~Hz}, 1 \mathrm{H}), 7.67(\mathrm{~d}, J=8.4 \mathrm{~Hz}, 1 \mathrm{H}), 7.65-7.63(\mathrm{~m}$, 1H), 7.57 (s, 1H), 7.51-7.47 (m, 2H)), $7.41(\mathrm{~d}, J=4.8 \mathrm{~Hz}, 2 \mathrm{H}), 7.31(\mathrm{~s}, 3 \mathrm{H}), 2.37$ (s, $3 \mathrm{H}) ;{ }^{13} \mathrm{C}$ NMR $\left(150 \mathrm{MHz}, \mathrm{CDCl}_{3}\right) \delta=150.8,140.3,137.9,134.7,133.3,131.9,131.1$, $130.7,130.2$, 129.5, 128.8, 128.5, 128.3, 128.0, 119.0, 118.6, 117.7, 113.5, 112.8, 11.8 ; HRMS (ESI) m/z calcd for $\mathrm{C}_{24} \mathrm{H}_{17} \mathrm{~N}_{4}{ }^{+}(\mathrm{M}+\mathrm{H})^{+} 361.1448$, found 361.1450.<smiles>N#Cc1ccc(-c2cn(-c3ccc(C#N)cc3)nc2-c2ccccc2)cc1</smiles>

4,4'-(3-phenyl-1H-pyrazole-1,4-diyl)dibenzonitrile (3e):

Yield: 80\% (139.8 mg); yellow solid; IR (KBr): 3443, 2361, 2335, 2220, 1649, 1602, 1512, 1397, 1343, 1222, $841 \mathrm{~cm}^{-1} ;{ }^{1} \mathrm{H}$ NMR (600 MHz, $\left.\mathrm{CDCl}_{3}\right) \delta=8.18(\mathrm{~s}, 1 \mathrm{H}), 7.94$ $(\mathrm{d}, J=8.4 \mathrm{~Hz}, 2 \mathrm{H}), 7.79(\mathrm{~d}, J=9.0 \mathrm{~Hz}, 2 \mathrm{H}), 7.62(\mathrm{~d}, J=8.4 \mathrm{~Hz}, 2 \mathrm{H}), 7.52(\mathrm{~d}, J=5.4$ $\mathrm{Hz}, 2 \mathrm{H}), 7.44(\mathrm{~d}, J=8.4 \mathrm{~Hz}, 2 \mathrm{H}), 7.39(\mathrm{~d}, J=6.6 \mathrm{~Hz}, 3 \mathrm{H}) ;{ }^{13} \mathrm{C}$ NMR $(150 \mathrm{MHz}$, $\left.\mathrm{CDCl}_{3}\right) \delta=151.9,142.2,136.9,133.7,132.4,131.6,128.9,128.8,128.6,128.4,126.8$, 122.4, 118.7, 118.2, 110.8, 109.9; HRMS (ESI) m/z calcd for $\mathrm{C}_{23} \mathrm{H}_{15} \mathrm{~N}_{4}{ }^{+}(\mathrm{M}+\mathrm{H})^{+}$ 347.1291 , found 347.1282 .<smiles>CC(=O)c1ccc(-c2cn(-c3ccc(C(C)=O)cc3)nc2-c2ccccc2)cc1</smiles>

dimethyl 4,4'-(3-phenyl-1H-pyrazole-1,4-diyl)dibenzoate (3f): 
Yield: 78\% (160.1 mg); white solid; IR (KBr): 3441, 2361, 2335, 1718, 1605, 1520, $1435,1278,1224,1110,857 \mathrm{~cm}^{-1} ;{ }^{1} \mathrm{H}$ NMR $\left(600 \mathrm{MHz}, \mathrm{CDCl}_{3}\right) \delta=8.16-8.15(\mathrm{~m}$, $3 \mathrm{H}), 8.00(\mathrm{~d}, J=7.8 \mathrm{~Hz}, 2 \mathrm{H}), 7.88(\mathrm{~d}, J=9.0 \mathrm{~Hz}, 2 \mathrm{H}), 7.56-7.55(\mathrm{~m}, 2 \mathrm{H}), 7.40(\mathrm{~d}, J$ $=8.4 \mathrm{~Hz}, 2 \mathrm{H}), 7.37-7.36(\mathrm{~m}, 3 \mathrm{H}), 3.94(\mathrm{~s}, 3 \mathrm{H}), 3.92(\mathrm{~s}, 3 \mathrm{H}) ;{ }^{13} \mathrm{C}$ NMR $(150 \mathrm{MHz}$, $\left.\mathrm{CDCl}_{3}\right) \delta=166.8,166.3,151.5,142.7,137.1,132.2,131.1,129.8,128.6,128.4,128.3$, 127.9, 126.8(1), 126.8, 122.7, 118.0, 52.2, 52.1; HRMS (ESI) $\mathrm{m} / \mathrm{z}$ calcd for $\mathrm{C}_{25} \mathrm{H}_{21} \mathrm{~N}_{2} \mathrm{O}_{4}^{+}(\mathrm{M}+\mathrm{H})^{+}$413.1496, found 413.1495.<smiles>CCOC(=O)c1ccc(-c2cn(-c3ccc(C(=O)OCC)cc3)nc2-c2ccccc2)cc1</smiles>

$3 g$

diethyl 4,4'-(3-phenyl-1H-pyrazole-1,4-diyl)dibenzoate (3g):

Yield: 76\% (167.1 mg); white solid; IR (KBr): 3444, 2361, 2336, 1711, 1607, 1519, $1401,1367,1273,1106,856 \mathrm{~cm}^{-1} ;{ }^{1} \mathrm{H}$ NMR $\left(600 \mathrm{MHz}, \mathrm{CDCl}_{3} / \mathrm{DMSO}-d_{6}\right) \delta=8.47(\mathrm{~s}$, $1 \mathrm{H}), 8.13(\mathrm{~d}, J=8.4 \mathrm{~Hz}, 2 \mathrm{H}), 7.98-7.96(\mathrm{~m}, 4 \mathrm{H}), 7.54-7.53(\mathrm{~m}, 2 \mathrm{H}), 7.43(\mathrm{~d}, J=$ $8.4 \mathrm{~Hz}, 2 \mathrm{H}), 7.36(\mathrm{~d}, J=4.2 \mathrm{~Hz}, 3 \mathrm{H}), 4.40-4.34(\mathrm{~m}, 4 \mathrm{H}), 1.43-1.38(\mathrm{~m}, 6 \mathrm{H}) ;{ }^{13} \mathrm{C}$ NMR $\left(150 \mathrm{MHz}, \mathrm{CDCl}_{3} / \mathrm{DMSO}-d_{6}\right) \delta=165.5,165.0,150.6,142.2,136.6,131.9$, 130.5, 130.4, 129.2, 129.1, 128.3, 127.9, 127.8, 127.7, 127.5, 122.0, 117.4, 60.5, 60.3, 13.8; HRMS (ESI) $\mathrm{m} / \mathrm{z}$ calcd for $\mathrm{C}_{27} \mathrm{H}_{25} \mathrm{~N}_{2} \mathrm{O}_{4}{ }^{+}(\mathrm{M}+\mathrm{H})^{+} 441.1809$, found 441.1805 .<smiles>CCCCOC(=O)c1ccc(-c2cn(-c3ccc(C(=O)OCC)cc3)nc2-c2ccccc2)cc1</smiles>

\section{di-tert-butyl 4,4'-(3-phenyl-1H-pyrazole-1,4-diyl)dibenzoate $(3 \mathrm{~h})$ :}

Yield: 71\% (176.1 mg); white solid; IR (KBr): 3442, 2959, 2361, 1712, 1607, 1520, $1277,1230,1175,1111,855 \mathrm{~cm}^{-1} ;{ }^{1} \mathrm{H}$ NMR $\left(400 \mathrm{MHz}, \mathrm{CDCl}_{3}\right) \delta=8.17(\mathrm{~d}, J=7.6$ $\mathrm{Hz}, 3 \mathrm{H}), 8.01(\mathrm{~d}, J=8.0 \mathrm{~Hz}, 2 \mathrm{H}), 7.89(\mathrm{~d}, J=8.4 \mathrm{~Hz}, 2 \mathrm{H}), 7.57-7.55(\mathrm{~m}, 2 \mathrm{H}), 7.41$ $(\mathrm{d}, J=8.4 \mathrm{~Hz}, 2 \mathrm{H}), 7.37-7.36(\mathrm{~m}, 3 \mathrm{H}), 4.37-4.32(\mathrm{~m}, 4 \mathrm{H}), 1.82-1.71(\mathrm{~m}, 4 \mathrm{H})$, $1.55-1.44(\mathrm{~m}, 4 \mathrm{H}), 1.02-0.97(\mathrm{~m}, 6 \mathrm{H}) ;{ }^{13} \mathrm{C} \mathrm{NMR}\left(100 \mathrm{MHz}, \mathrm{CDCl}_{3}\right) \delta=166.4,165.9$, $151.5,142.7,137.1,132.4,131.1,131.0,129.8,129.1,128.5(1)$ 128.5, 128.4, 128.3, 126.8, 122.8, 118.1, 65.0, 64.9, 30.8, 28.2, 19.3, 13.8; HRMS (ESI) m/z calcd for $\mathrm{C}_{31} \mathrm{H}_{33} \mathrm{~N}_{2} \mathrm{O}_{4}{ }^{+}(\mathrm{M}+\mathrm{H})^{+}$497.2435, found 497.2434. 
<smiles>COc1ccc(-n2nc(-c3ccccc3)c(-c3ccc(S(=O)(=O)OC)cc3)c2C)cc1</smiles>

$3 \mathbf{i}$

5-methyl-1,4-bis(4-(methylsulfonyl)phenyl)-3-phenyl-1H-pyrazole (3i):

Yield: 75\% (174.5 mg); light yellow solid; IR (KBr): 3441, 2361, 1592, 1497, 1309, 1150, 1091, 1029, 958, $846 \mathrm{~cm}^{-1}$; ${ }^{1} \mathrm{H}$ NMR (400 MHz, DMSO) $\delta=8.15(\mathrm{~d}, J=8.8 \mathrm{~Hz}$, $2 \mathrm{H}), 8.02-7.97(\mathrm{~m}, 4 \mathrm{H}), 7.52(\mathrm{~d}, J=8.4 \mathrm{~Hz}, 2 \mathrm{H}), 7.42-7.40(\mathrm{~m}, 2 \mathrm{H}), 7.37-7.35(\mathrm{~m}$, $3 \mathrm{H}), 3.33(\mathrm{~s}, 3 \mathrm{H}), 3.28(\mathrm{~s}, 3 \mathrm{H}), 2.41(\mathrm{~s}, 3 \mathrm{H}) ;{ }^{13} \mathrm{C} \mathrm{NMR}\left(150 \mathrm{MHz}, \mathrm{CDCl}_{3}\right) \delta=151.2$, $143.8,139.3,139.1,138.9,138.2$, 131.9, 130.9, 128.7, 128.5, 128.4, 128.2, 127.6, 125.0, 119.8, 44.6, 44.4, 12.2; HRMS (ESI) m/z calcd for $\mathrm{C}_{24} \mathrm{H}_{23} \mathrm{~N}_{2} \mathrm{O}_{4} \mathrm{~S}_{2}{ }^{+}(\mathrm{M}+\mathrm{H})^{+}$ 467.1094, found 467.1090.<smiles>Fc1ccc(-c2cn(-c3ccc(F)cc3)nc2-c2ccccc2)cc1</smiles>

\section{1,4-bis(4-fluorophenyl)-3-phenyl-1H-pyrazole (3j):}

Yield: 61\% (101.1 mg); light yellow oil; IR (KBr): 3443, 2361, 1648, 1506, 1451, 1396, 1220, 837, $801 \mathrm{~cm}^{-1} ;{ }^{1} \mathrm{H}$ NMR $\left(600 \mathrm{MHz}, \mathrm{CDCl}_{3}\right) \delta=7.93(\mathrm{~s}, 1 \mathrm{H}), 7.76-7.74$ $(\mathrm{m}, 2 \mathrm{H}), 7.56-7.55(\mathrm{~m}, 2 \mathrm{H}), 7.35-7.33(\mathrm{~m}, 3 \mathrm{H}), 7.30-7.28(\mathrm{~m}, 2 \mathrm{H}), 7.17(\mathrm{t}, J=8.4$ $\mathrm{Hz}, 2 \mathrm{H}), 7.03(\mathrm{t}, J=9.0 \mathrm{~Hz}, 2 \mathrm{H}) ;{ }^{13} \mathrm{C} \mathrm{NMR}\left(150 \mathrm{MHz}, \mathrm{CDCl}_{3}\right) \delta=162.0(\mathrm{~d}, J=$ $\left.245.1 \mathrm{~Hz},{ }^{1} J_{\mathrm{CF}}\right), 161.1\left(\mathrm{~d}, J=244.7 \mathrm{~Hz},{ }^{1} J_{\mathrm{CF}}\right), 150.4,136.2,130.3\left(\mathrm{~d}, J=7.8 \mathrm{~Hz},{ }^{3} J_{\mathrm{CF}}\right)$, $130.2,128.3\left(\mathrm{~d}, J=9.6 \mathrm{~Hz},{ }^{3} J_{\mathrm{CF}}\right), 128.0,126.6,122.0,120.7,120.6,116.2(\mathrm{~d}, J=23.0$ $\left.\mathrm{Hz},{ }^{2} J_{\mathrm{CF}}\right), 116.0,115.5\left(\mathrm{~d}, J=21.3 \mathrm{~Hz},{ }^{2} J_{\mathrm{CF}}\right.$ ); HRMS (ESI) $\mathrm{m} / \mathrm{z}$ calcd for $\mathrm{C}_{21} \mathrm{H}_{15} \mathrm{~F}_{2} \mathrm{~N}_{2}{ }^{+}$ $(\mathrm{M}+\mathrm{H})^{+} 333.1198$, found 333.1198.<smiles>Clc1ccccc1-c1cn(-c2ccccc2Cl)nc1-c1ccccc1</smiles>

3k

1,4-bis(2-chlorophenyl)-3-phenyl-1H-pyrazole (3k): 
Yield: 58\% (105.4 mg); yellow solid; IR (KBr): 3444, 2361, 2336, 1647, 1554, 1452 , 1397, $1041 \mathrm{~cm}^{-1}$; ${ }^{1} \mathrm{H}$ NMR (600 MHz, $\left.\mathrm{CDCl}_{3}\right) \delta=8.07(\mathrm{~s}, 1 \mathrm{H}), 7.79(\mathrm{~d}, J=7.8 \mathrm{~Hz}$, $1 \mathrm{H}), 7.55-7.51(\mathrm{~m}, 3 \mathrm{H}), 7.48(\mathrm{~d}, J=7.8 \mathrm{~Hz}, 1 \mathrm{H}), 7.43-7.41(\mathrm{~m}, 1 \mathrm{H}), 7.35(\mathrm{~d}, J=$ $7.2 \mathrm{~Hz}, 2 \mathrm{H}), 7.31(\mathrm{~s}, 2 \mathrm{H}), 7.25-7.22(\mathrm{~m}, 2 \mathrm{H}) ;{ }^{13} \mathrm{C} \mathrm{NMR}\left(150 \mathrm{MHz}, \mathrm{CDCl}_{3}\right) \delta=150.8$, 137.9, 133.9, 132.8, 132.7, 132.5, 131.9, 130.7, 129.9, 128.9, 128.8, 128.3, 127.9, 127.8, 127.7, 126.7, 118.3; HRMS (ESI) m/z calcd for $\mathrm{C}_{21} \mathrm{H}_{15} \mathrm{Cl}_{2} \mathrm{~N}_{2}{ }^{+}(\mathrm{M}+\mathrm{H})^{+}$ 365.0607 , found 365.0609 .<smiles>Clc1ccc(-c2cn(-c3ccc(Cl)cc3)nc2-c2ccccc2)cc1</smiles>

3I

\section{1,4-bis(4-chlorophenyl)-3-phenyl-1H-pyrazole (3l):}

Yield: 65\% (118.1 mg); gray solid; IR (KBr): 3443, 2361, 1649, 1492, 1446, 1394, 1352, 1221, 1105, $833 \mathrm{~cm}^{-1} ;{ }^{1} \mathrm{H}$ NMR $\left(600 \mathrm{MHz}, \mathrm{CDCl}_{3}\right) \delta=7.94(\mathrm{~s}, 1 \mathrm{H}), 7.71(\mathrm{~d}, J=$ $7.8 \mathrm{~Hz}, 2 \mathrm{H}), 7.54$ (d, $J=4.8 \mathrm{~Hz}, 2 \mathrm{H}), 7.42$ (d, $J=7.8 \mathrm{~Hz}, 2 \mathrm{H}), 7.34$ (s, 3H), 7.29 (d, $J$ $=7.2 \mathrm{~Hz}, 2 \mathrm{H}), 7.24(\mathrm{~d}, J=7.2 \mathrm{~Hz}, 2 \mathrm{H}) ;{ }^{13} \mathrm{C} \mathrm{NMR}\left(150 \mathrm{MHz}, \mathrm{CDCl}_{3}\right) \delta=150.7,138.2$, 132.9, 132.5, 131.9, 131.0, 129.8, 129.5, 128.7, 128.4, 128.3, 128.2, 126.4, 122.0, 119.9; HRMS (ESI) $\mathrm{m} / \mathrm{z}$ calcd for $\mathrm{C}_{21} \mathrm{H}_{15} \mathrm{Cl}_{2} \mathrm{~N}_{2}{ }^{+}(\mathrm{M}+\mathrm{H})^{+}$365.0607, found 365.0609.<smiles>Brc1ccc(-c2cn(-c3ccc(Br)cc3)nc2-c2ccccc2)cc1</smiles>

\section{1,4-bis(4-bromophenyl)-3-phenyl-1H-pyrazole (3m):}

Yield: 70\% (158.0 mg); light yellow solid; IR (KBr): 3442, 1639, 1547, 1447, 1394, 1266, 1222, 1068, $831 \mathrm{~cm}^{-1}$; ${ }^{1} \mathrm{H}$ NMR $\left(600 \mathrm{MHz}, \mathrm{CDCl}_{3}\right) \delta=7.98(\mathrm{~s}, 1 \mathrm{H}), 7.68(\mathrm{~d}, J=$ $8.4 \mathrm{~Hz}, 2 \mathrm{H}), 7.59$ (d, $J=8.4 \mathrm{~Hz}, 2 \mathrm{H}), 7.55(\mathrm{~d}, J=3.0 \mathrm{~Hz}, 2 \mathrm{H}), 7.46(\mathrm{~d}, J=8.4 \mathrm{~Hz}$, $2 \mathrm{H}), 7.35(\mathrm{~s}, 3 \mathrm{H}), 7.20(\mathrm{~d}, J=7.8 \mathrm{~Hz}, 2 \mathrm{H}) ;{ }^{13} \mathrm{C} \mathrm{NMR}\left(150 \mathrm{MHz}, \mathrm{CDCl}_{3}\right) \delta=150.7$, 138.7, 135.1, 132.5, 131.7, 131.4, 130.2, 128.5, 128.4, 128.2, 126.3, 122.1, 121.1, 120.3, 119.7; HRMS (ESI) $\mathrm{m} / \mathrm{z}$ calcd for $\mathrm{C}_{21} \mathrm{H}_{15} \mathrm{Br}_{2} \mathrm{~N}_{2}{ }^{+}(\mathrm{M}+\mathrm{H})^{+} 452.9597$, found 452.9596. 
<smiles>Ic1ccc(-c2cn(-c3ccc(I)cc3)nc2-c2ccccc2)cc1</smiles>

\section{1,4-bis(4-iodophenyl)-3-phenyl-1H-pyrazole (3n):}

Yield: 55\% (150.6 mg); white solid; IR (KBr): 3443, 1640, 1448, 1392, 1264, 1028, $1003,828,769,671 \mathrm{~cm}^{-1} ;{ }^{1} \mathrm{H}$ NMR $\left(600 \mathrm{MHz}, \mathrm{DMSO}-d_{6}\right) \delta=8.84(\mathrm{~s}, 1 \mathrm{H}), 7.88(\mathrm{~d}, J$ $=4.8 \mathrm{~Hz}, 2 \mathrm{H}), 7.78-7.76(\mathrm{~m}, 2 \mathrm{H}), 7.73-7.71(\mathrm{~m}, 2 \mathrm{H}), 7.47(\mathrm{~d}, J=5.4 \mathrm{~Hz}, 2 \mathrm{H})$, $7.40(\mathrm{~d}, J=4.8 \mathrm{~Hz}, 3 \mathrm{H}), 7.15-7.13(\mathrm{~m}, 2 \mathrm{H}) ;{ }^{13} \mathrm{C} \mathrm{NMR}\left(150 \mathrm{MHz}, \mathrm{CDCl}_{3}\right) \delta=149.7$, $139.0,138.2$, 137.4, 132.5, 131.8, 130.3, 128.6, 128.3, 128.2, 128.1, 121.4, 120.3, 93.1, 91.4; HRMS (ESI) $\mathrm{m} / \mathrm{z}$ calcd for $\mathrm{C}_{21} \mathrm{H}_{15} \mathrm{I}_{2} \mathrm{~N}_{2}{ }^{+}(\mathrm{M}+\mathrm{H})^{+}$548.9319, found 548.9311.<smiles>COC(=O)c1ccsc1-c1cn(-c2sccc2C(C)=O)nc1-c1ccccc1</smiles>

30

dimethyl 2,2'-(3-phenyl-1H-pyrazole-1,4-diyl)bis(thiophene-3-carboxylate) (3o):

Yield: 52\% (110.1 mg); light yellow oil; IR (KBr): 3441, 1670, 1548, 1438, 1401, 1267, 1071, 878, $772 \mathrm{~cm}^{-1} ;{ }^{1} \mathrm{H}$ NMR $\left(600 \mathrm{MHz}, \mathrm{CDCl}_{3}\right) \delta=8.63(\mathrm{~s}, 1 \mathrm{H}), 7.63(\mathrm{~d}, J=$ $5.4 \mathrm{~Hz}, 1 \mathrm{H}), 7.54(\mathrm{~d}, J=5.4 \mathrm{~Hz}, 1 \mathrm{H}), 7.51-7.49(\mathrm{~m}, 2 \mathrm{H}), 7.42(\mathrm{~d}, J=4.8 \mathrm{~Hz}, 1 \mathrm{H})$, $7.31-7.29(\mathrm{~m}, 3 \mathrm{H}), 6.90(\mathrm{~d}, J=4.8 \mathrm{~Hz}, 1 \mathrm{H}), 3.88(\mathrm{~s}, 3 \mathrm{H}), 3.70(\mathrm{~s}, 3 \mathrm{H}) ;{ }^{13} \mathrm{C} \mathrm{NMR}$ $\left(150 \mathrm{MHz}, \mathrm{CDCl}_{3}\right) \delta=162.5,161.3,151.5,142.5,138.4,134.4,132.8,132.2,130.5$, 130.0, 128.2, 128.0, 127.9, 127.8, 126.9, 118.3, 114.7, 52.4, 51.9; HRMS (ESI) m/z calcd for $\mathrm{C}_{21} \mathrm{H}_{17} \mathrm{~N}_{2} \mathrm{O}_{4} \mathrm{~S}_{2}{ }^{+}(\mathrm{M}+\mathrm{H})^{+} 425.0624$, found 425.0627 .<smiles>CCOC(=O)c1ccc(-c2nn(-c3ccc(C(=O)OCC)cc3)cc2-c2ccc(C)cc2)cc1</smiles>

diethyl 4,4'-(3-(p-tolyl)-1H-pyrazole-1,4-diyl)dibenzoate (4a): 
Yield: 72\% (163.1 mg); white solid; IR (KBr): 3441, 1715, 1647, 1445, 1398, 1272 , $1173,1107,830 \mathrm{~cm}^{-1} ;{ }^{1} \mathrm{H}$ NMR $\left(600 \mathrm{MHz}, \mathrm{CDCl}_{3}\right) \delta=8.16-8.14(\mathrm{~m}, 3 \mathrm{H}), 8.01(\mathrm{~d}, J$ $=8.4 \mathrm{~Hz}, 2 \mathrm{H}), 7.87(\mathrm{~d}, J=8.4 \mathrm{~Hz}, 2 \mathrm{H}), 7.45-7.40(\mathrm{~m}, 4 \mathrm{H}), 7.17(\mathrm{~d}, J=7.8 \mathrm{~Hz}, 2 \mathrm{H})$, $4.41-4.37(\mathrm{~m}, 4 \mathrm{H}), 2.38(\mathrm{~s}, 3 \mathrm{H}), 1.45-1.33(\mathrm{~m}, 6 \mathrm{H}) ;{ }^{13} \mathrm{C} \mathrm{NMR}\left(150 \mathrm{MHz}, \mathrm{CDCl}_{3}\right)$ $\delta=166.4,165.8,151.6,142.7,138.3,137.1,131.1,129.8,129.4,129.2,128.9$, 128.3(3), 128.3, 128.1, 126.7, 122.6, 117.9, 61.1, 61.0, 21.3, 14.3; HRMS (ESI) m/z calcd for $\mathrm{C}_{28} \mathrm{H}_{27} \mathrm{~N}_{2} \mathrm{O}_{4}{ }^{+}(\mathrm{M}+\mathrm{H})^{+} 455.1965$, found 455.1969 .<smiles>CCOC(=O)c1ccc(-c2cn(-c3ccc(C(=O)OCC)cc3)nc2-c2ccccc2OC)cc1</smiles>

4b

diethyl 4,4'-(3-(2-methoxyphenyl)-1H-pyrazole-1,4-diyl)dibenzoate (4b):

Yield: 70\% (164.3 mg); yellow solid; IR (KBr): 3441, 1709, 1607, 1519, 1464, 1403, 1274, 1177, 1021, $857 \mathrm{~cm}^{-1} ;{ }^{1} \mathrm{H}$ NMR $\left(600 \mathrm{MHz} \mathrm{CDCl}_{3}\right) \delta=8.24(\mathrm{~s}, 1 \mathrm{H}), 8.15(\mathrm{~d}, J=$ $7.8 \mathrm{~Hz}, 2 \mathrm{H}), 7.94(\mathrm{~d}, J=7.8 \mathrm{~Hz}, 2 \mathrm{H}), 7.87(\mathrm{~d}, J=7.8 \mathrm{~Hz}, 2 \mathrm{H}), 7.59$ (d, $J=6.6 \mathrm{~Hz}$, $1 \mathrm{H}), 7.41(\mathrm{t}, J=7.2 \mathrm{~Hz}, 1 \mathrm{H}), 7.31(\mathrm{~d}, J=7.8 \mathrm{~Hz}, 2 \mathrm{H}), 7.08(\mathrm{t}, J=7.2 \mathrm{~Hz}, 1 \mathrm{H}), 6.87(\mathrm{~d}$, $J=7.8 \mathrm{~Hz}, 1 \mathrm{H}), 4.41-4.35(\mathrm{~m}, 4 \mathrm{H}), 3.35(\mathrm{~s}, 3 \mathrm{H}), 1.43-1.37(\mathrm{~m}, 6 \mathrm{H}) ;{ }^{13} \mathrm{C} \mathrm{NMR}$ $\left(150 \mathrm{MHz}, \mathrm{CDCl}_{3}\right) \delta=166.5,165.9,157.0,149.7,142.8,138.0,131.2,131.1,130.4$, 129.6, 128.4, 128.1, 126.5, 125.5, 124.1, 121.9, 120.9, 118.0, 111.1, 61.1, 60.9, 54.9, 14.3; HRMS (ESI) m/z calcd for $\mathrm{C}_{28} \mathrm{H}_{27} \mathrm{~N}_{2} \mathrm{O}_{5}{ }^{+}(\mathrm{M}+\mathrm{H})^{+} 471.1915$, found 471.1915.<smiles>CCOC(=O)c1ccc(-c2cn(-c3ccc(C(=O)OCC)cc3)nc2-c2ccc(F)cc2)cc1</smiles>

diethyl 4,4'-(3-(4-fluorophenyl)-1H-pyrazole-1,4-diyl)dibenzoate (4c):

Yield: 61\% (139.1 mg); yellow solid; IR (KBr): 3440, 1711, 1608, 1441, 1342, 1281, 1107, 1020, 842, $768 \mathrm{~cm}^{-1} ;{ }^{1} \mathrm{H}$ NMR $\left(600 \mathrm{MHz}, \mathrm{CDCl}_{3}\right) \delta=8.18-8.15(\mathrm{~m}, 3 \mathrm{H}), 8.03$ $(\mathrm{d}, J=8.4 \mathrm{~Hz}, 2 \mathrm{H}), 7.88(\mathrm{~d}, J=8.4 \mathrm{~Hz}, 2 \mathrm{H}), 7.55-7.53(\mathrm{~m}, 2 \mathrm{H}), 7.40(\mathrm{~d}, J=8.4 \mathrm{~Hz}$, $2 \mathrm{H}), 7.06(\mathrm{t}, J=9.0 \mathrm{~Hz}, 2 \mathrm{H}), 4.43-4.38(\mathrm{~m}, 4 \mathrm{H}), 1.44-1.40(\mathrm{~m}, 6 \mathrm{H}) ;{ }^{13} \mathrm{C} \mathrm{NMR}(150$ $\left.\mathrm{MHz}, \mathrm{CDCl}_{3}\right) \delta=166.3,165.8,162.9\left(\mathrm{~d}, J=246.9 \mathrm{~Hz},{ }^{1} J_{\mathrm{CF}}\right), 150.6,142.6,136.8$, $131.1,130.3\left(\mathrm{~d}, J=7.8 \mathrm{~Hz},{ }^{3} J_{\mathrm{CF}}\right), 129.9,129.2,128.4,128.3,126.9,122.7,118.0$, $115.5\left(\mathrm{~d}, J=21.5 \mathrm{~Hz},{ }^{2} J_{\mathrm{CF}}\right), 61.2,61.0,14.3$; HRMS (ESI) $\mathrm{m} / \mathrm{z}$ calcd for $\mathrm{C}_{27} \mathrm{H}_{24} \mathrm{FN}_{2} \mathrm{O}_{4}{ }^{+}(\mathrm{M}+\mathrm{H})^{+} 459.1715$, found 459.1702 . 
<smiles>CCOC(=O)c1ccc(-c2cn(-c3ccc(C(=O)OCC)cc3)nc2-c2ccc(Cl)cc2)cc1</smiles>

diethyl 4,4'-(3-(4-chlorophenyl)-1H-pyrazole-1,4-diyl)dibenzoate (4d):

Yield: 63\% (149.1 mg); white solid; IR (KBr): 3441, 1707, 1637, 1447, 1398, 1278 , 1228, 1106, $599 \mathrm{~cm}^{-1} ;{ }^{1} \mathrm{H}$ NMR $\left(600 \mathrm{MHz}, \mathrm{CDCl}_{3}\right) \delta=8.18-8.15(\mathrm{~m}, 3 \mathrm{H}), 8.03(\mathrm{~d}, J$ $=7.8 \mathrm{~Hz}, 2 \mathrm{H}), 7.88(\mathrm{~d}, J=8.4 \mathrm{~Hz}, 2 \mathrm{H}), 7.50(\mathrm{~d}, J=8.4 \mathrm{~Hz}, 2 \mathrm{H}), 7.40(\mathrm{~d}, J=7.8 \mathrm{~Hz}$, $2 \mathrm{H}), 7.33(\mathrm{~d}, J=8.4 \mathrm{~Hz}, 2 \mathrm{H}), 4.43-4.39(\mathrm{~m}, 4 \mathrm{H}), 1.44-1.40(\mathrm{~m}, 6 \mathrm{H}) ;{ }^{13} \mathrm{C} \mathrm{NMR}$ $\left(150 \mathrm{MHz}, \mathrm{CDCl}_{3}\right) \delta=166.3,165.8,150.3,142.6,136.7,134.4,131.2,130.8,129.9$, 129.7, 129.3, 128.7, 128.5, 128.4, 127.0, 122.8, 118.1, 61.2, 61.1, 14.4; HRMS (ESI) $\mathrm{m} / \mathrm{z}$ calcd for $\mathrm{C}_{27} \mathrm{H}_{24} \mathrm{ClN}_{2} \mathrm{O}_{4}{ }^{+}(\mathrm{M}+\mathrm{H})^{+} 475.1419$, found 475.1422 .<smiles>CCOC(=O)c1ccc(-c2c(-c3ccccc3)nn(-c3ccc(C(=O)OCC)cc3)c2C)cc1</smiles>

diethyl 4,4' -(5-methyl-3-phenyl-1H-pyrazole-1,4-diyl)dibenzoate (4e):

Yield: 82\% (186.1 mg); yellow solid; IR (KBr): 3441, 1711, 1604, 1511, 1442, 1367, $1272,1174,1020,864,700 \mathrm{~cm}^{-1} ;{ }^{1} \mathrm{H}$ NMR $\left(600 \mathrm{MHz}, \mathrm{CDCl}_{3}\right) \delta=8.20(\mathrm{~d}, J=7.8 \mathrm{~Hz}$, $2 \mathrm{H}), 8.06(\mathrm{~d}, J=7.2 \mathrm{~Hz}, 2 \mathrm{H}), 7.70(\mathrm{~d}, J=7.8 \mathrm{~Hz}, 2 \mathrm{H}), 7.47(\mathrm{~s}, 2 \mathrm{H}), 7.34(\mathrm{~d}, J=7.8$ $\mathrm{Hz}, 2 \mathrm{H}), 7.28(\mathrm{~s}, 3 \mathrm{H}), 4.43-4.39(\mathrm{~m}, 4 \mathrm{H}), 2.38(\mathrm{~s}, 3 \mathrm{H}), 1.44-1.41(\mathrm{~m}, 6 \mathrm{H}) ;{ }^{13} \mathrm{C}$ NMR $\left(150 \mathrm{MHz}, \mathrm{CDCl}_{3}\right) \delta=166.5,165.8,150.6,143.2,138.3,137.8,132.5,130.6$, 130.1, 129.7, 129.4, 128.9, 128.3, 128.2, 127.9, 124.2, 120.2, 61.2, 61.0, 14.3, 12.1; HRMS (ESI) $\mathrm{m} / \mathrm{z}$ calcd for $\mathrm{C}_{28} \mathrm{H}_{27} \mathrm{~N}_{2} \mathrm{O}_{4}{ }^{+}(\mathrm{M}+\mathrm{H})^{+} 455.1965$, found 455.1955.<smiles>CCOC(=O)c1ccc(-c2c(-c3ccccc3)nn(-c3ccc(C(=O)OCC)cc3)c2-c2ccccc2)cc1</smiles>

diethyl 4,4'-(3,5-diphenyl-1H-pyrazole-1,4-diyl)dibenzoate (4f): 
Yield: 60\% (154.8 mg); gray solid; IR (KBr): 3437, 1715, 1604, 1446, 1274, 1173 , $1101,1019,965,855,698 \mathrm{~cm}^{-1} ;{ }^{1} \mathrm{H}$ NMR $\left(400 \mathrm{MHz} \mathrm{CDCl}_{3}\right) \delta=7.99(\mathrm{~d}, J=8.4 \mathrm{~Hz}$, $2 \mathrm{H}), 7.89(\mathrm{~d}, J=8.0 \mathrm{~Hz}, 2 \mathrm{H}), 7.50-7.49(\mathrm{~m}, 2 \mathrm{H}), 7.39(\mathrm{~d}, J=8.4 \mathrm{~Hz}, 2 \mathrm{H}), 7.30(\mathrm{~d}, J$ $=5.2 \mathrm{~Hz}, 4 \mathrm{H}), 7.26(\mathrm{~d}, J=8.0 \mathrm{~Hz}, 2 \mathrm{H}), 7.15(\mathrm{~d}, J=8.0 \mathrm{~Hz}, 2 \mathrm{H}), 7.07(\mathrm{~d}, J=7.2 \mathrm{~Hz}$, $2 \mathrm{H}), 4.49-4.32(\mathrm{~m}, 4 \mathrm{H}), 1.39-1.35(\mathrm{~m}, 6 \mathrm{H}) ;{ }^{13} \mathrm{C} \mathrm{NMR}\left(100 \mathrm{MHz}, \mathrm{CDCl}_{3}\right) \delta=166.4$, $165.8,150.9,143.1,141.7,137.6,136.8,133.0,132.4,130.4,130.2(2), 130.2$, 129.4, 128.9, 128.7(3), 128.7, 128.6, 128.5, 128.4, 128.3, 128.1, 127.4, 124.4, 120.5, 61.1, 60.9, 14.3; HRMS (ESI) $\mathrm{m} / \mathrm{z}$ calcd for $\mathrm{C}_{33} \mathrm{H}_{29} \mathrm{~N}_{2} \mathrm{O}_{4}{ }^{+}(\mathrm{M}+\mathrm{H})^{+}$517.2122, found 517.2124 .<smiles>CCOC(=O)c1ccc(-c2c(-c3ccc(C)cc3)c(-c3ccccc3O)nn2-c2ccc(C(=O)OCC)cc2)cc1</smiles>

diethyl 4,4'-(3-(2-hydroxyphenyl)-5-phenyl-1H-pyrazole-1,4-diyl)dibenzoate (4g):

Yield: 48\% (127.5 mg); white solid; IR (KBr): 3411, 1712, 1605, 1415, 1275, 1172 , $1102,1019,975,857,760,697 \mathrm{~cm}^{-1} ;{ }^{1} \mathrm{H}$ NMR $\left(600 \mathrm{MHz}, \mathrm{CDCl}_{3}\right) \delta=7.91(\mathrm{~d}, J=7.8$ $\mathrm{Hz}, 2 \mathrm{H}), 7.82(\mathrm{~d}, J=7.8 \mathrm{~Hz}, 2 \mathrm{H}), 7.49(\mathrm{~d}, J=6.6 \mathrm{~Hz}, 2 \mathrm{H}), 7.45(\mathrm{~d}, J=7.8 \mathrm{~Hz}, 2 \mathrm{H})$, $7.29(\mathrm{~d}, J=6.0 \mathrm{~Hz}, 3 \mathrm{H}), 7.19-7.16(\mathrm{~m}, 3 \mathrm{H}), 6.97(\mathrm{~d}, J=7.2 \mathrm{~Hz}, 1 \mathrm{H}), 6.80-6.77(\mathrm{~m}$, $2 \mathrm{H}), 6.66(\mathrm{~s}, 1 \mathrm{H}), 4.32-4.28(\mathrm{~m}, 4 \mathrm{H}), 1.35-1.32(\mathrm{~m}, 6 \mathrm{H}) ;{ }^{13} \mathrm{C}$ NMR $(150 \mathrm{MHz}$, $\left.\mathrm{CDCl}_{3}\right) \delta=166.8,166.1,154.3,150.9,143.4,138.3,137.6,132.2,131.7,131.1,130.2$, $129.8,129.4,128.5(1), 128.5,128.4,128.3,128.1,123.4,121.4,120.6,116.5,116.1$, 61.2, 61.0, 14.2; HRMS (ESI) $\mathrm{m} / \mathrm{z}$ calcd for $\mathrm{C}_{33} \mathrm{H}_{29} \mathrm{~N}_{2} \mathrm{O}_{5}{ }^{+}(\mathrm{M}+\mathrm{H})^{+} 533.2071$, found 533.2072 .<smiles>CCOC(=O)c1ccc(-n2nc(-c3ccccc3O)c(-c3ccc(Cl)cc3)c2-c2ccc([SnH3])cc2)cc1</smiles>

diethyl 4,4'-(5-(4-chlorophenyl)-3-(2-hydroxyphenyl)-1H-pyrazole-1,4-diyl) dibenzoate (4h): 
Yield: 53\% (149.6 mg); yellow solid; IR (KBr): 3427, 1714, 1607, 1448, 1399, 1275, $1102,1016,859,756 \mathrm{~cm}^{-1}$; ${ }^{1} \mathrm{H}$ NMR $\left(400 \mathrm{MHz}, \mathrm{CDCl}_{3}\right) \delta=7.96(\mathrm{~d}, J=8.4 \mathrm{~Hz}, 2 \mathrm{H})$, $7.87(\mathrm{~d}, J=8.0 \mathrm{~Hz}, 2 \mathrm{H}), 7.45-7.43(\mathrm{~m}, 4 \mathrm{H}), 7.30-7.23(\mathrm{~m}, 4 \mathrm{H}), 7.16(\mathrm{~d}, J=8.0 \mathrm{~Hz}$, $2 \mathrm{H}), 6.98(\mathrm{~d}, J=7.6 \mathrm{~Hz}, 1 \mathrm{H}), 6.85-6.80(\mathrm{~m}, 2 \mathrm{H}), 4.37-4.31(\mathrm{~m}, 4 \mathrm{H}), 1.39-1.35(\mathrm{~m}$, $6 \mathrm{H}) ;{ }^{13} \mathrm{C} \mathrm{NMR}\left(100 \mathrm{MHz}, \mathrm{CDCl}_{3}\right) \delta=166.5,165.9,153.8,149.9,143.2,138.0,137.0$, $134.2,131.8,131.4,131.3,130.8,130.3,129.7,129.6,129.0,128.9,128.6,123.4$, $121.6,121.1,116.3,116.2,61.2,61.1,14.3$.<smiles></smiles>

\section{7,7'-(5-methyl-3-phenyl-1H-pyrazole-1,4-diyl)bis(4-methyl-2H-chromen-2-one)}

(4i):

Yield: 68\% (161.1 mg); yellow solid; IR (KBr): 3439, 1721, 1615, 1445, 1390, 1266, 1026, 944, 859, $701 \mathrm{~cm}^{-1}$; ${ }^{1} \mathrm{H}$ NMR (400 MHz, DMSO- $\left.d_{6}\right) \delta=7.88(\mathrm{~d}, J=8.4 \mathrm{~Hz}, 1 \mathrm{H})$, $7.71(\mathrm{~d}, J=8.0 \mathrm{~Hz}, 1 \mathrm{H}), 7.65(\mathrm{~d}, J=10.0 \mathrm{~Hz}, 2 \mathrm{H}), 7.35(\mathrm{~d}, J=4.0 \mathrm{~Hz}, 2 \mathrm{H}), 7.26(\mathrm{~s}$, $3 \mathrm{H}), 7.18(\mathrm{~s}, 1 \mathrm{H}), 7.12(\mathrm{~d}, J=8.0 \mathrm{~Hz}, 1 \mathrm{H}), 6.39-6.32(\mathrm{~m}, 2 \mathrm{H}), 2.41(\mathrm{~s}, 3 \mathrm{H}), 2.36(\mathrm{~s}$, $6 \mathrm{H}) ;{ }^{13} \mathrm{C}$ NMR $\left(100 \mathrm{MHz}, \mathrm{DMSO}-d_{6}\right) \delta=159.7,159.6,153.3,153.1,153.0,152.8$, $149.8,141.6,139.0,136.9,132.3,128.5,128.2$, 128.0, 126.4, 126.1, 125.6, 120.1, 119.0, 118.7, 118.4, 117.3, 114.5, 114.2, 111.7, 18.2, 18.1, 11.8; HRMS (ESI) m/z calcd for $\mathrm{C}_{30} \mathrm{H}_{23} \mathrm{~N}_{2} \mathrm{O}_{4}+(\mathrm{M}+\mathrm{H})^{+} 475.1652$, found 475.1642 .<smiles>c1ccc(NNc2ccccc2)cc1</smiles>

\section{1,2-diphenylhydrazine (7):}

Yield: $85 \%$ (78.1 mg); white solid; ${ }^{1} \mathrm{H}$ NMR $\left(600 \mathrm{MHz}, \mathrm{CDCl}_{3}\right) \delta=7.21(\mathrm{~s}, 4 \mathrm{H}), 6.83$ $(\mathrm{d}, J=4.8 \mathrm{~Hz}, 6 \mathrm{H}), 5.57(\mathrm{~s}, 2 \mathrm{H}) ;{ }^{13} \mathrm{C} \mathrm{NMR}\left(150 \mathrm{MHz}, \mathrm{CDCl}_{3}\right) \delta=148.8,129.3,119.8$, 112.2. The overall spectroscopic data are in complete agreement with assigned structures and consistent with literature. ${ }^{2}$ 
6. Copies of ${ }^{1} \mathrm{H}$ NMR, ${ }^{13} \mathrm{C}$ NMR

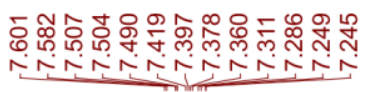

$\stackrel{\hat{m}}{i}$

\&
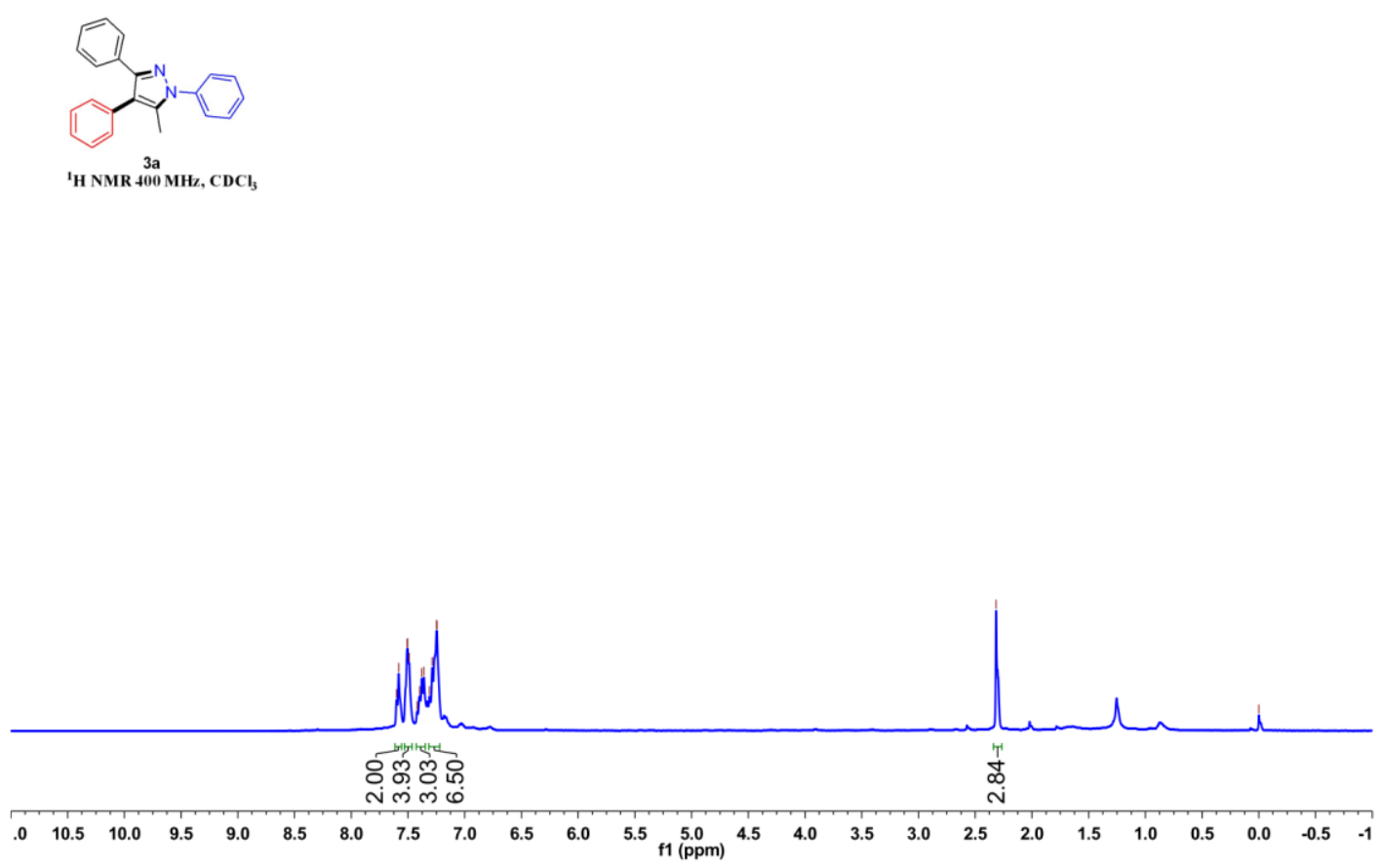

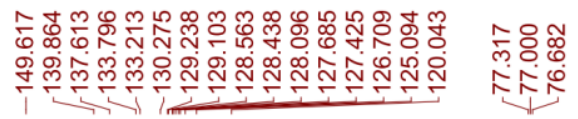

$\stackrel{+}{\stackrel{\leftrightarrow}{\circ}}$
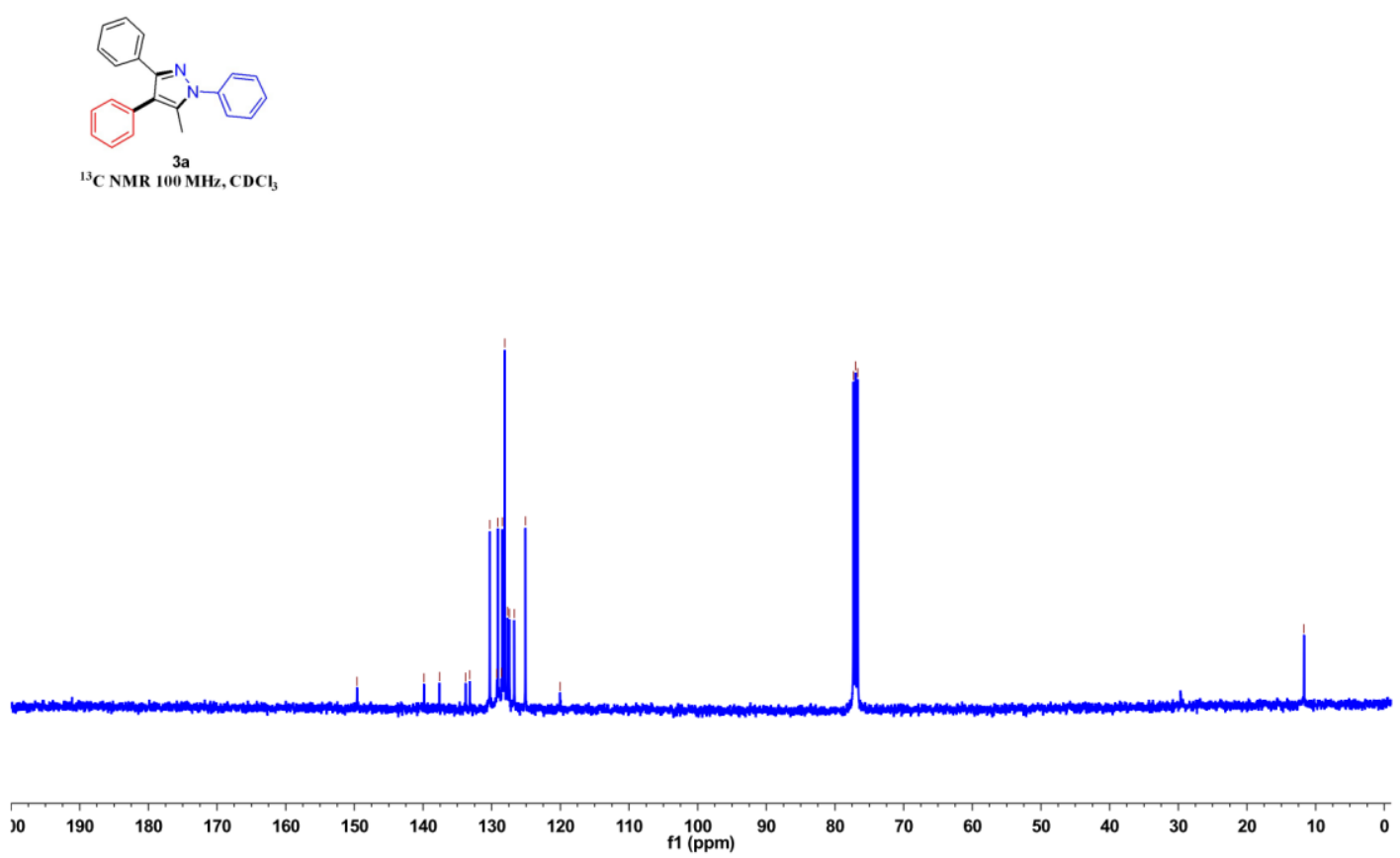

S16 

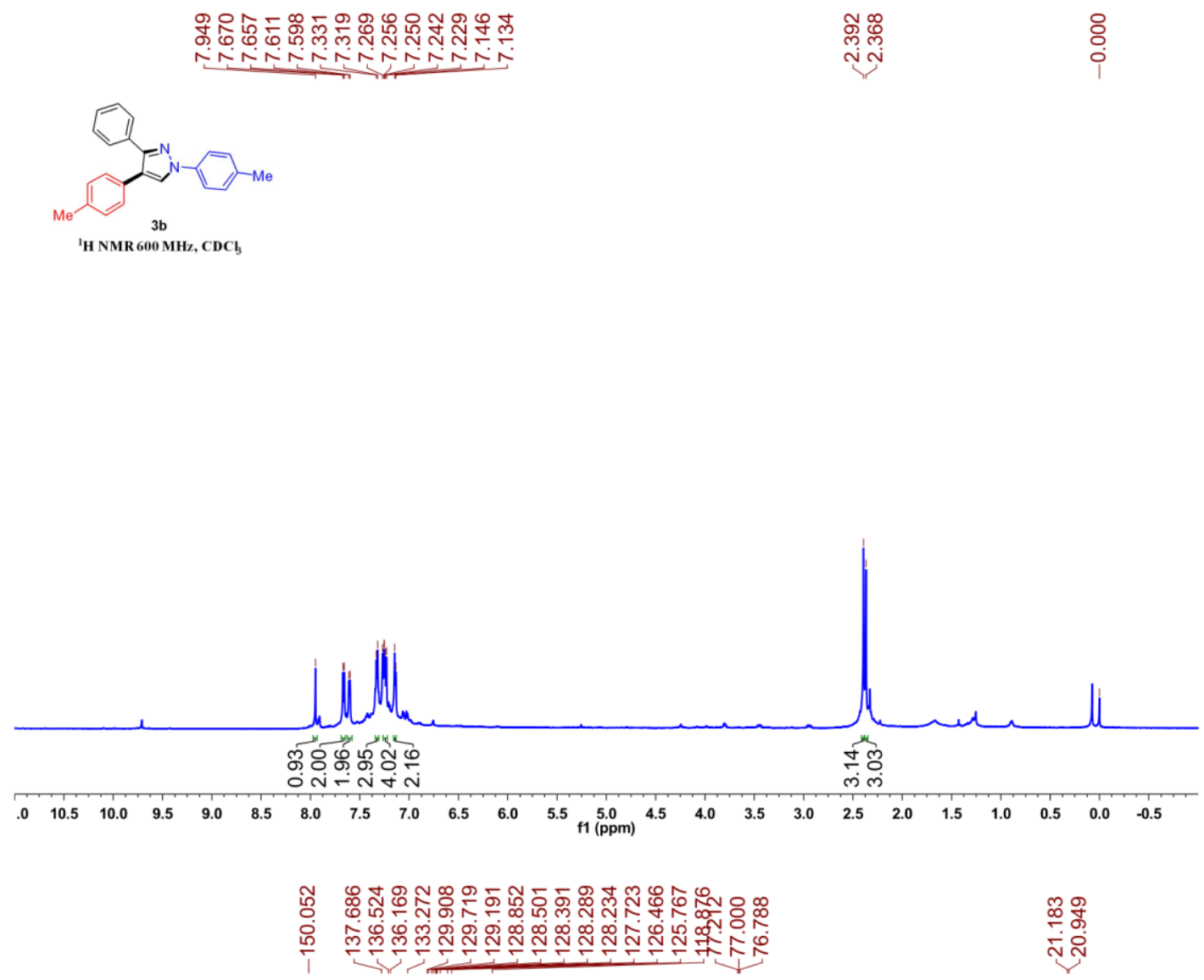

$\stackrel{\infty}{\infty} \stackrel{\substack{+ \\ \hdashline}}{\circ}$

ஸें
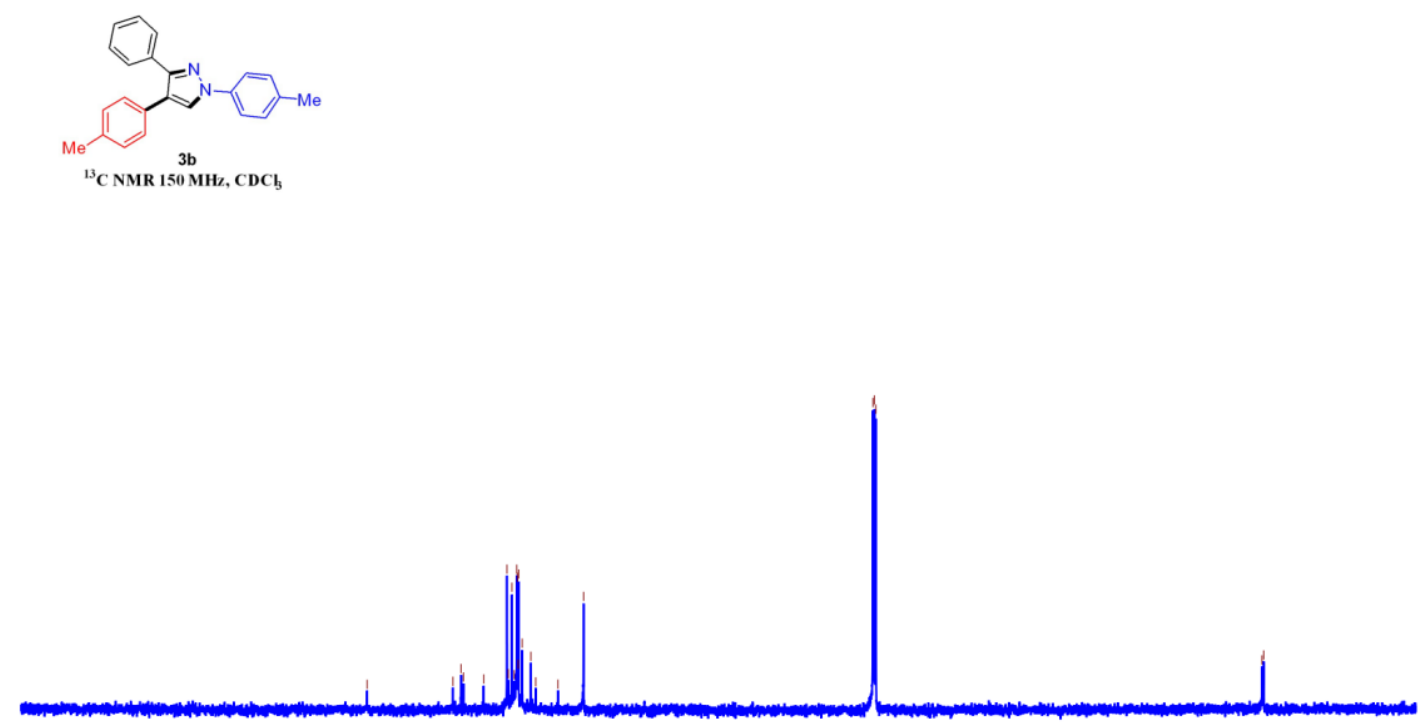

$\begin{array}{lllllllllllllllllllllllllll} & 190 & 180 & 170 & 160 & 150 & 140 & 130 & 120 & 110 & 100 & 90 & 80 & 70 & 60 & 50 & 40 & 30 & 20 & 10 & 0\end{array}$ 

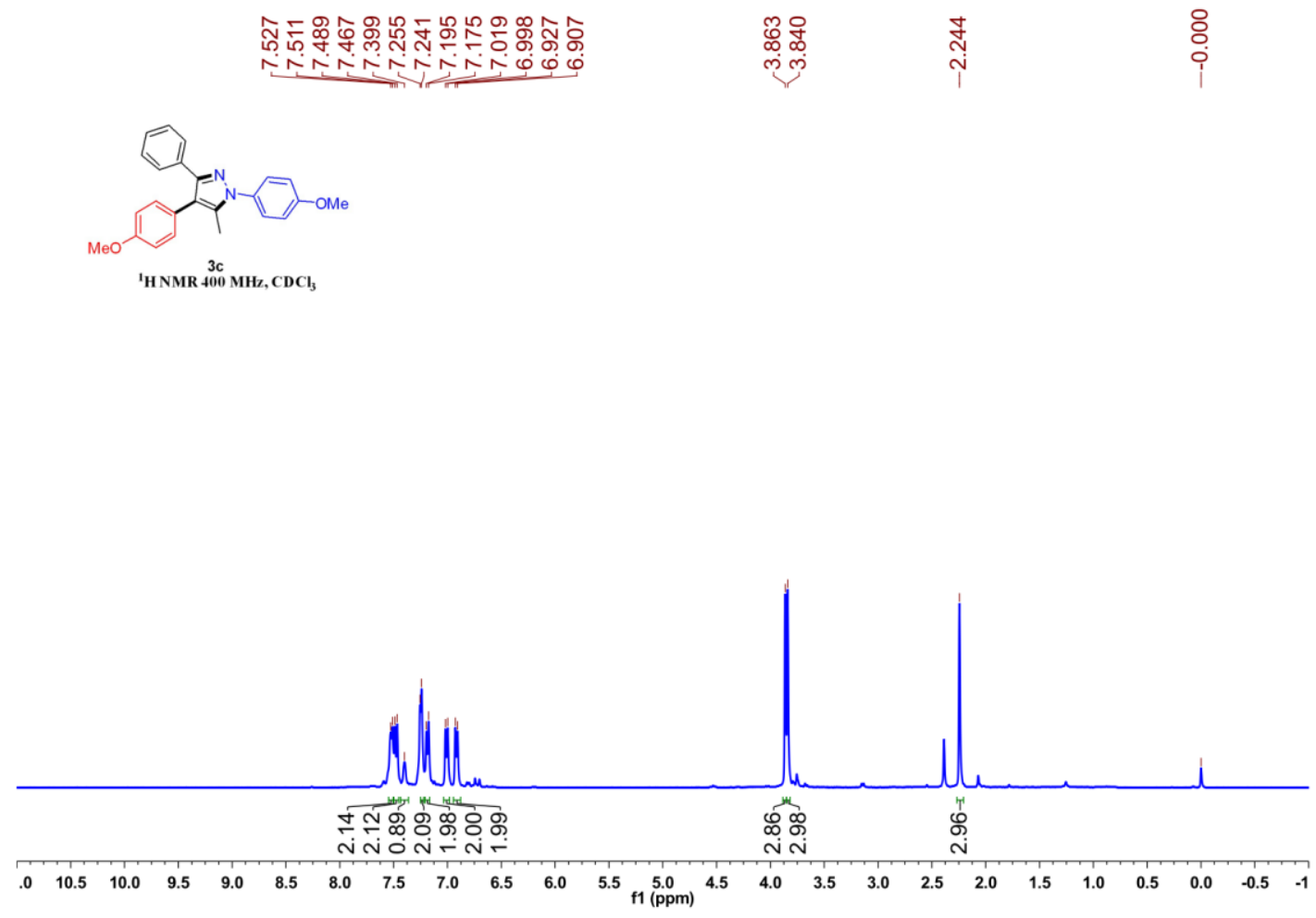

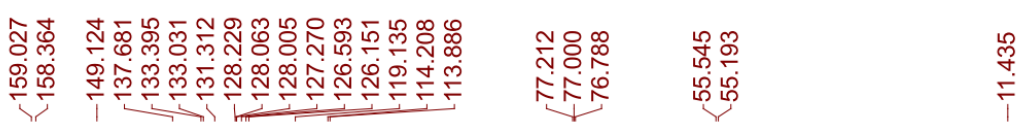
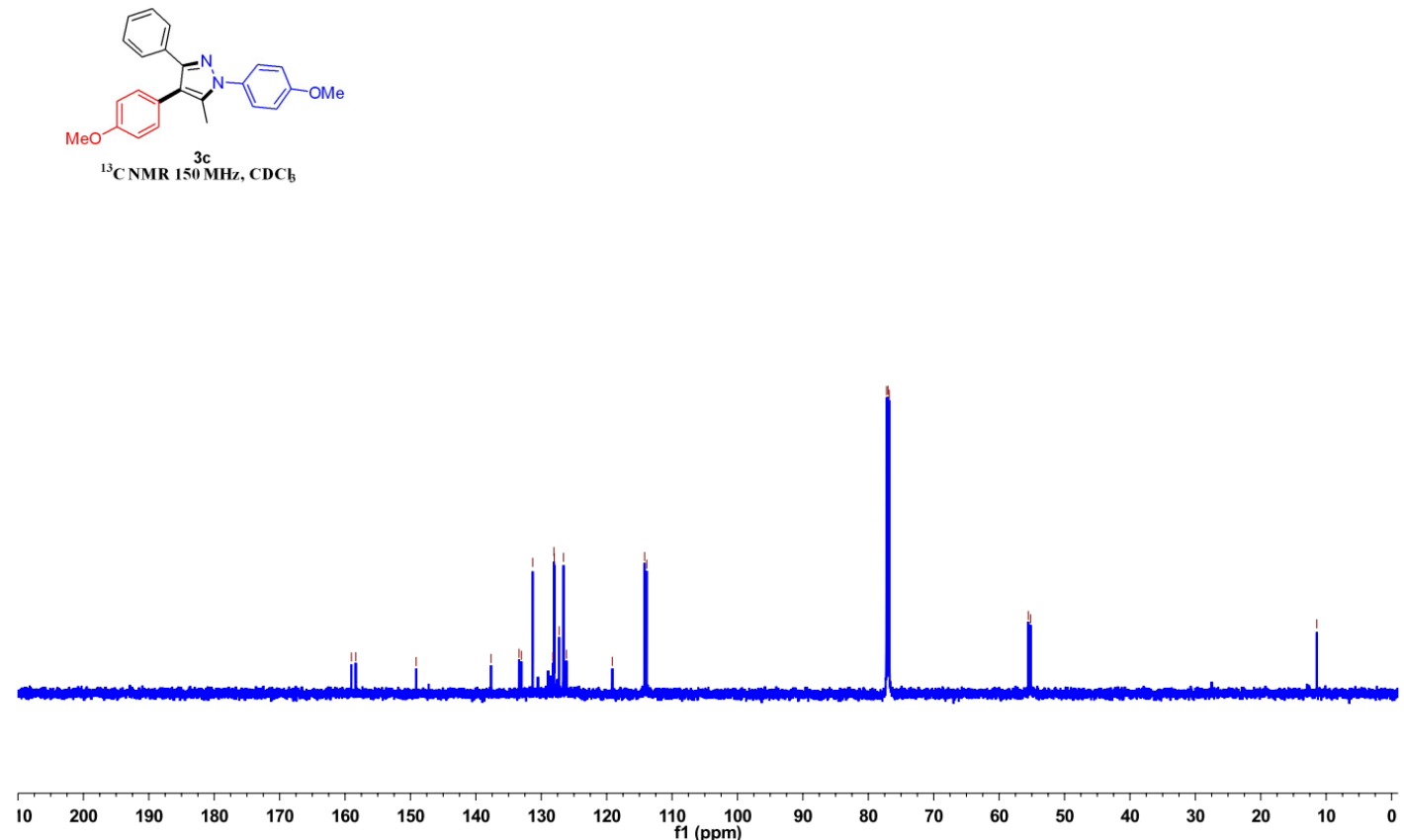

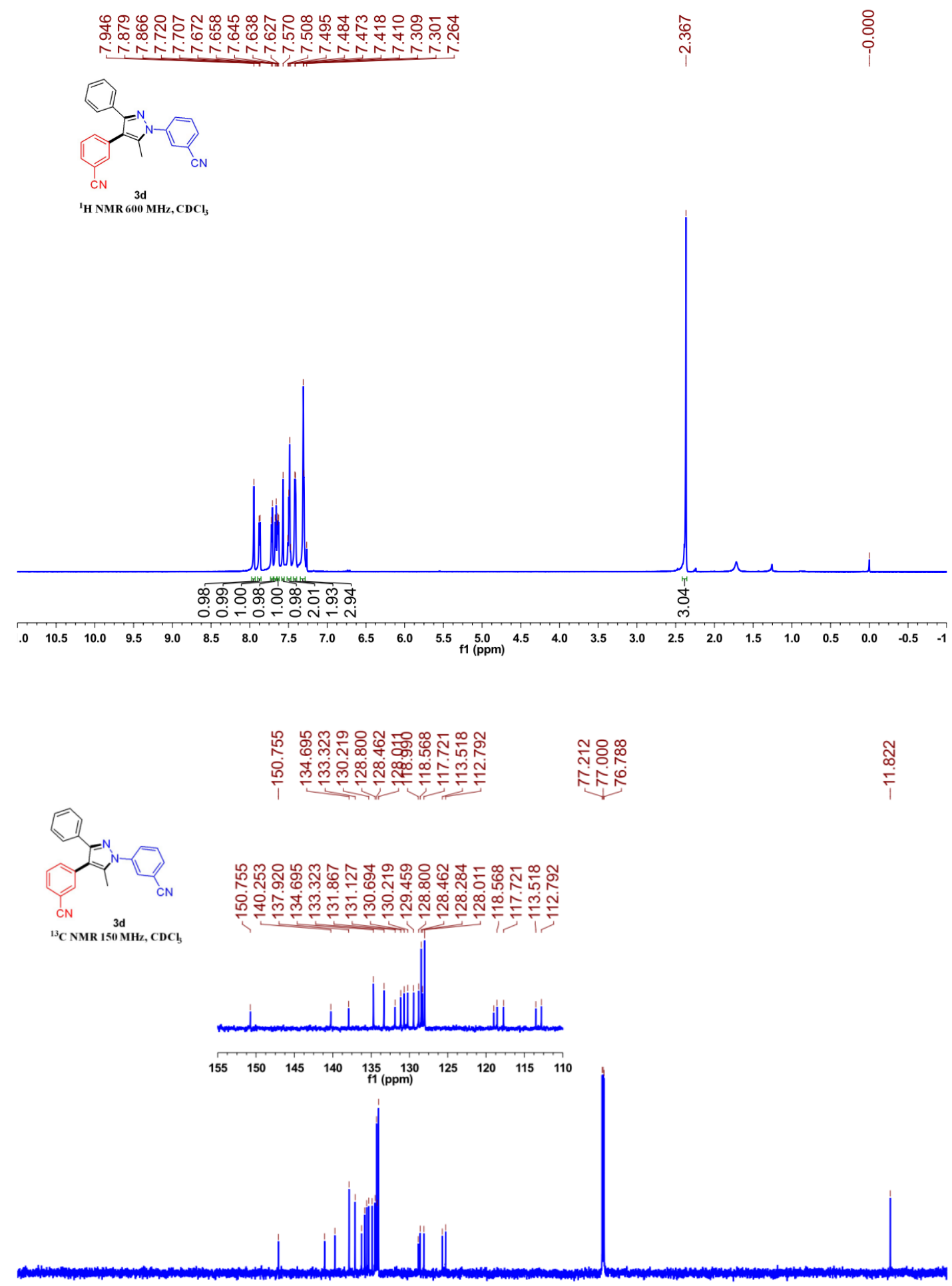

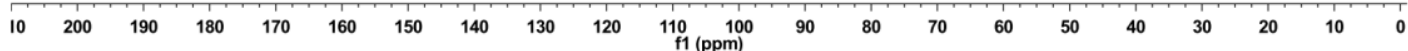



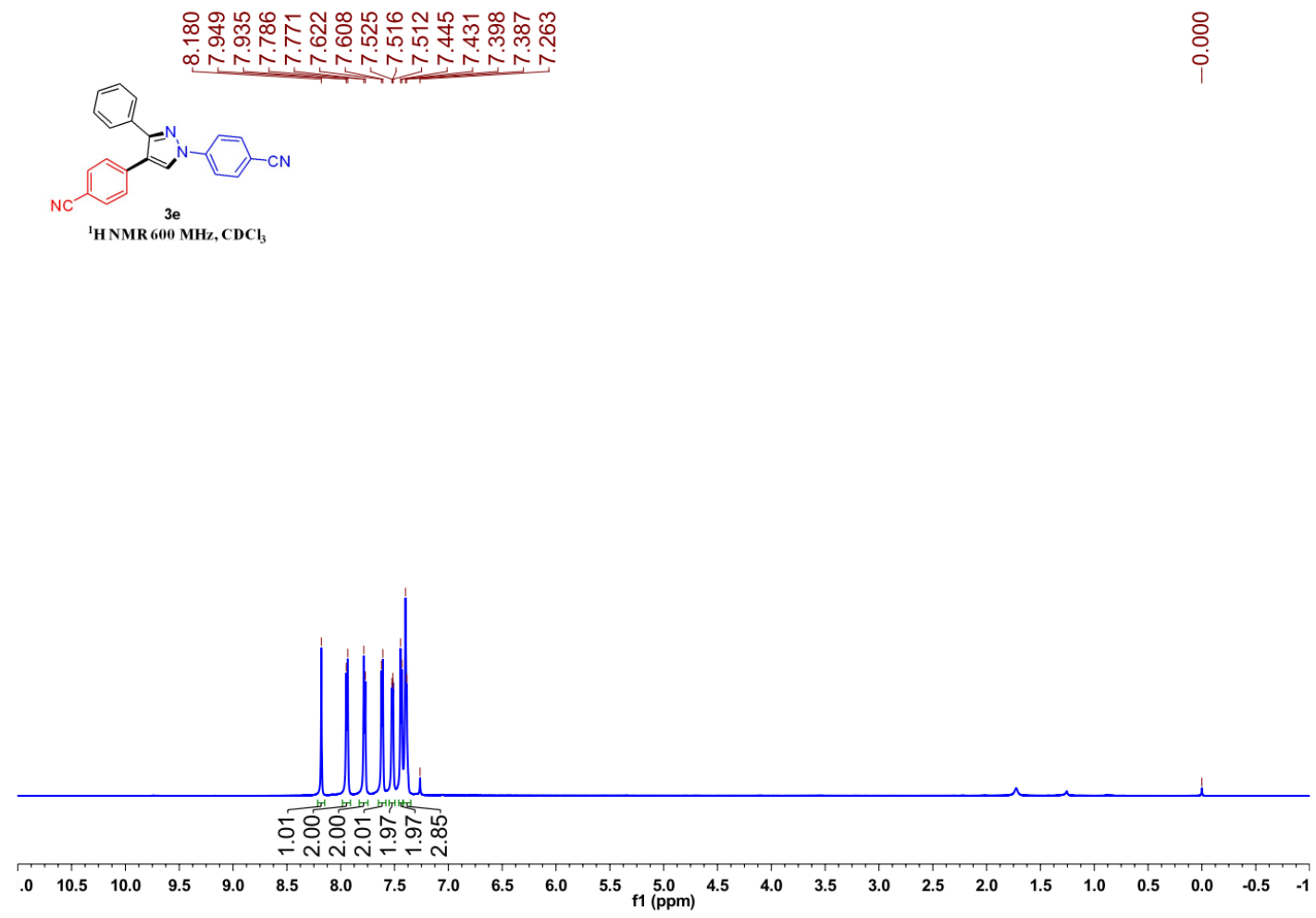

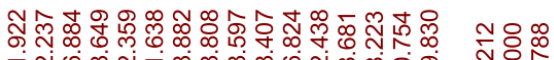

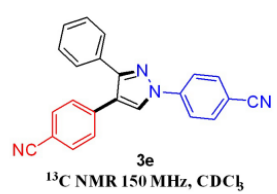

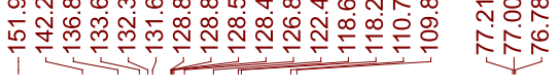

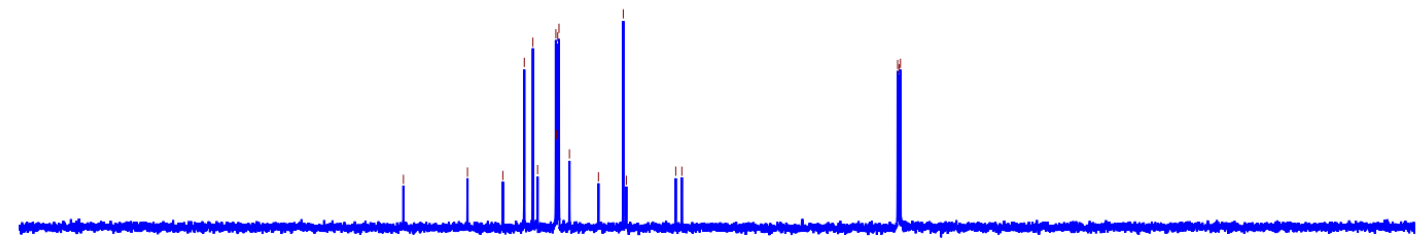

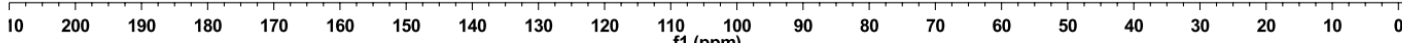




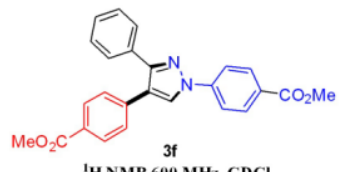

'H NMR $600 \mathrm{MHz}, \mathrm{CDCl}_{3}$

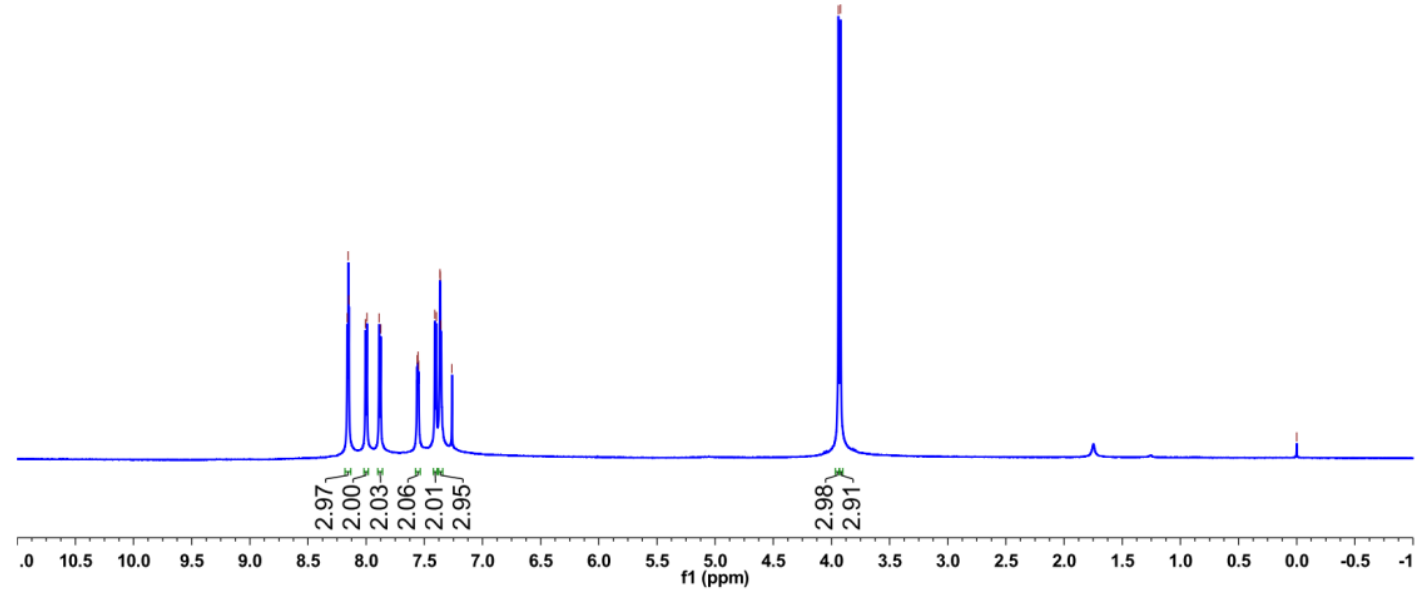

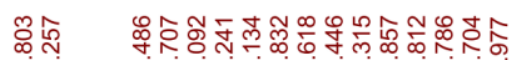

@ष

근

N命

ลิ ลิ

กิ่ กิ่
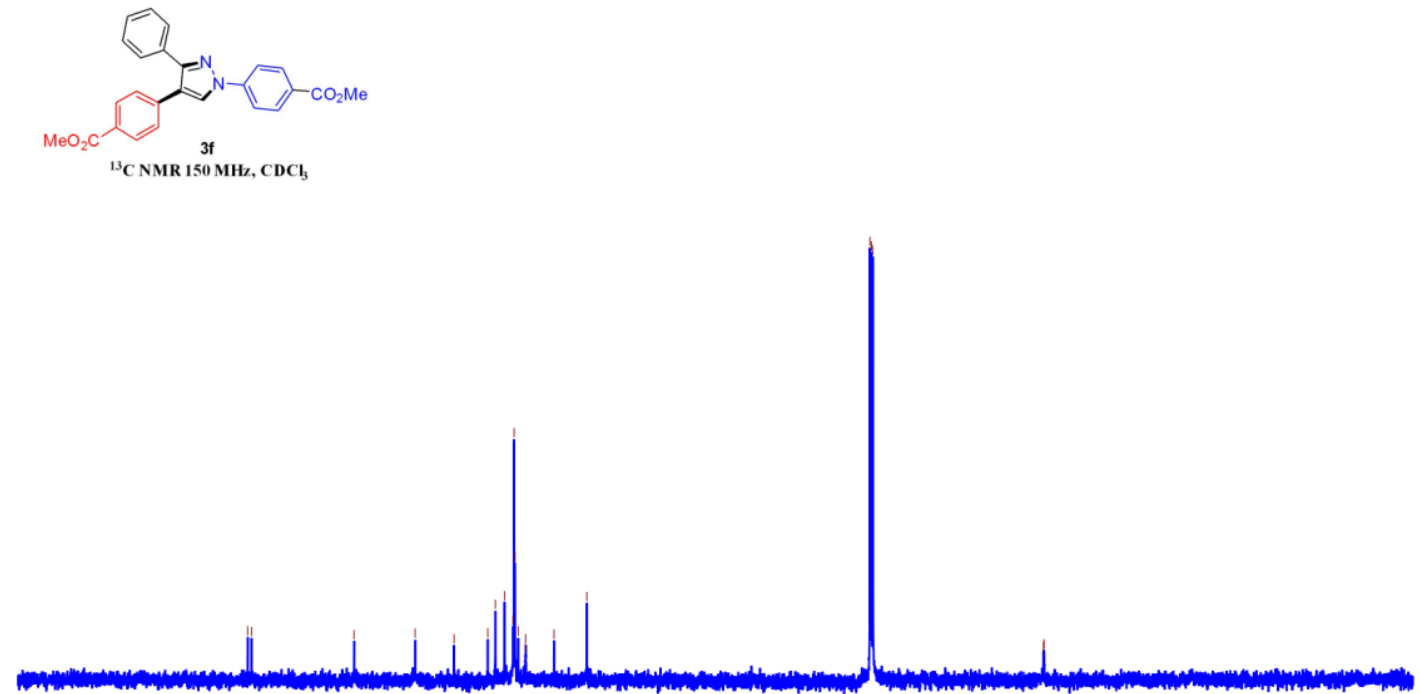

$\begin{array}{llllllllllllllllllllllllllll}30 & 190 & 180 & 170 & 160 & 150 & 140 & 130 & 120 & 110 & 100 & 90 & 80 & 70 & 60 & 50 & 40 & 30 & 20 & 10 & 0\end{array}$ 

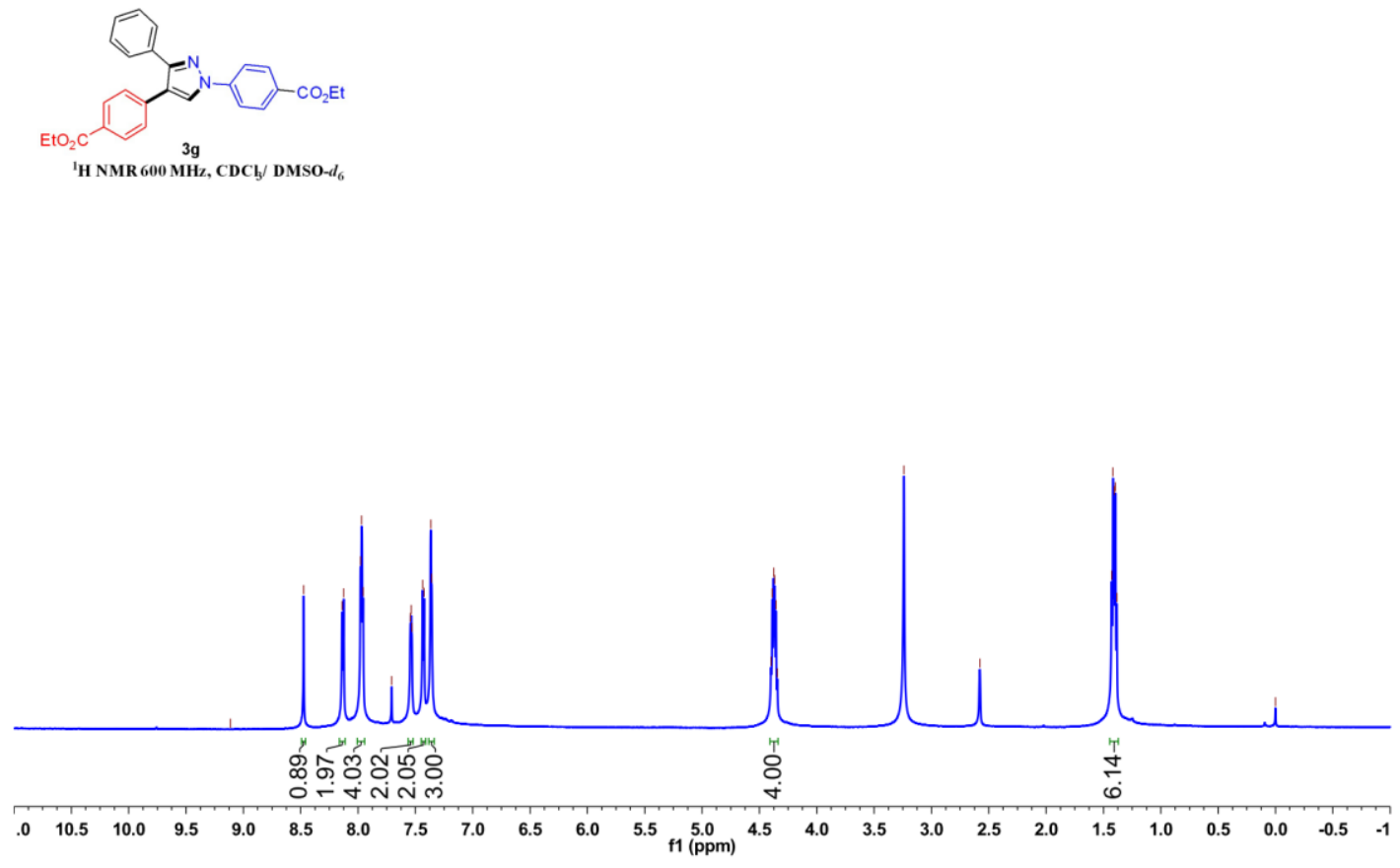

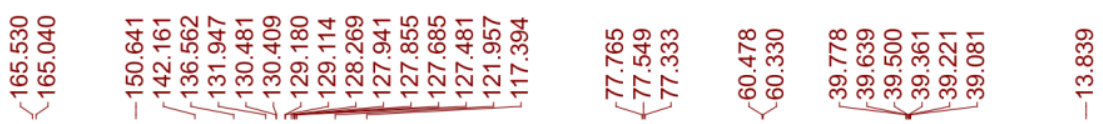<smiles></smiles>

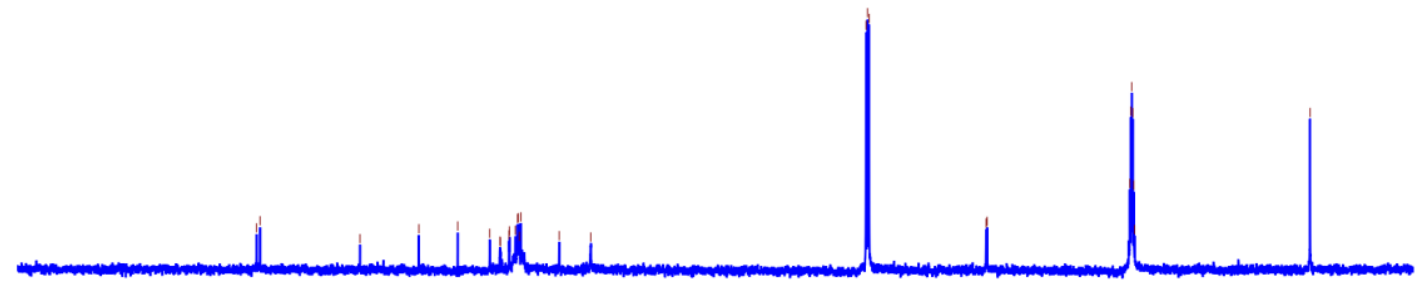

$\begin{array}{llllllllllllllllllllllllll} & 190 & 180 & 170 & 160 & 150 & 140 & 130 & 120 & 110 & 100 & 90 & 80 & 70 & 60 & 50 & 40 & 30 & 20 & 10 & 0\end{array}$ 

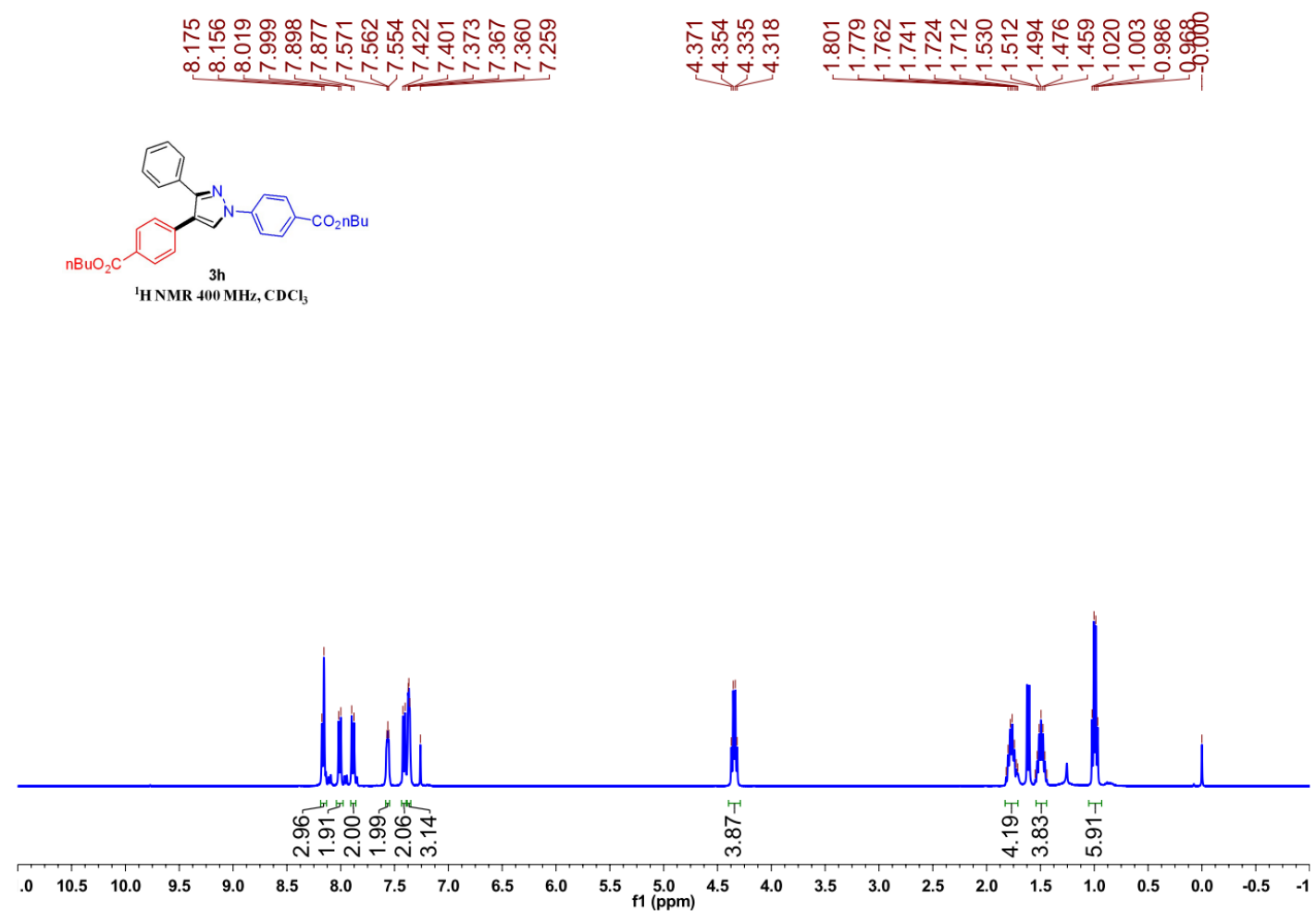

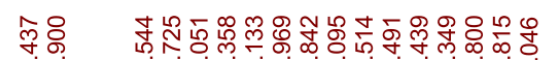

:

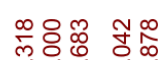

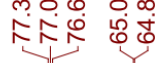

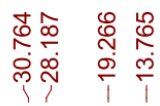

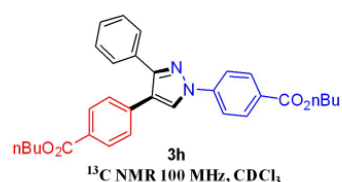

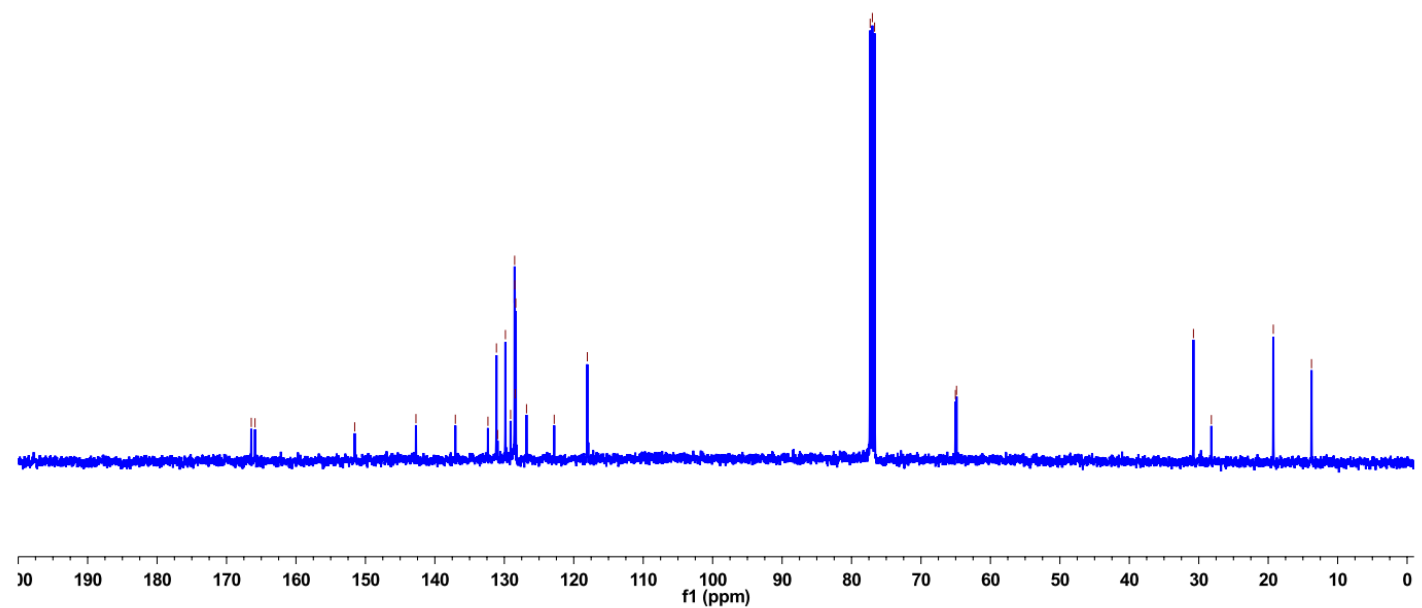



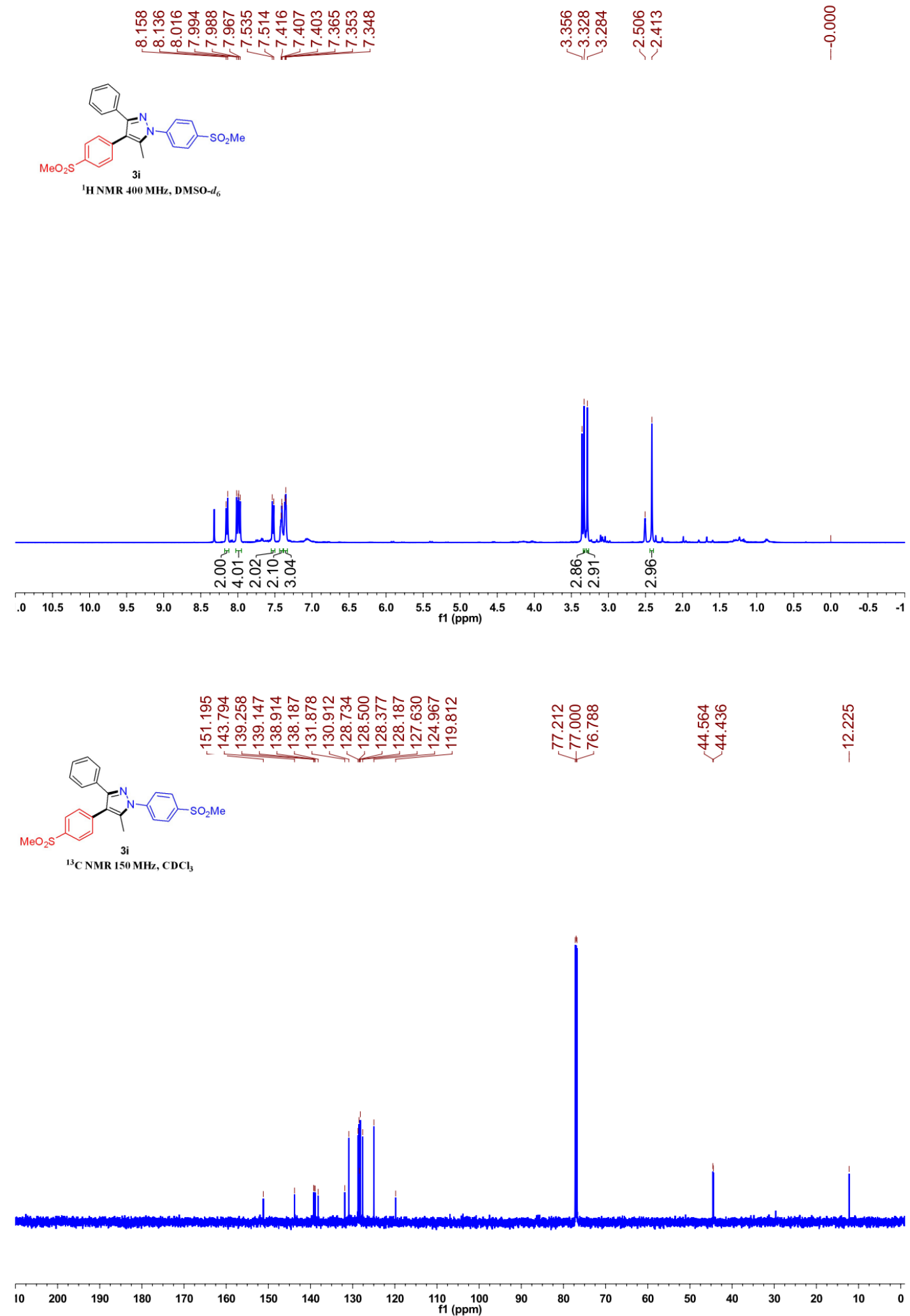

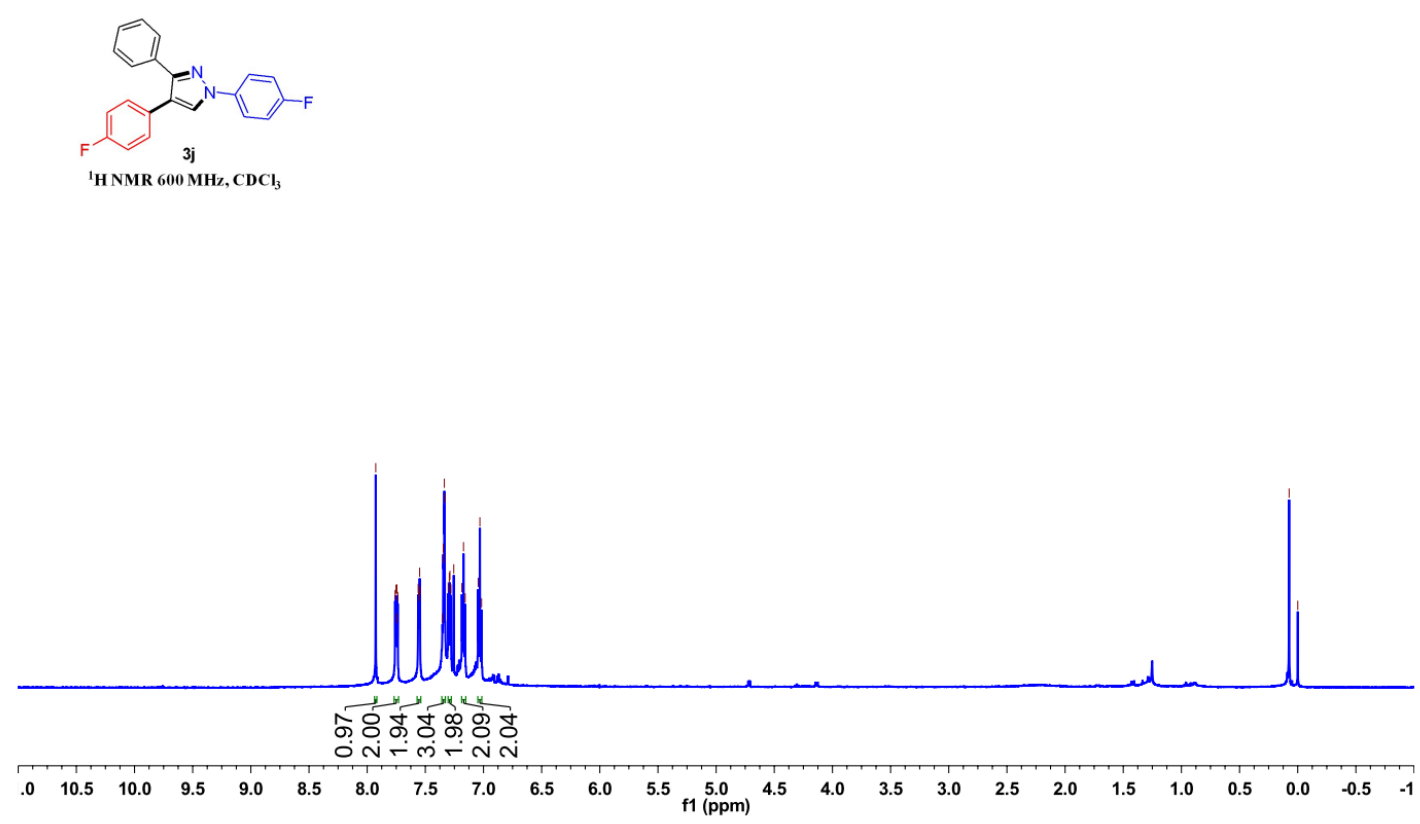

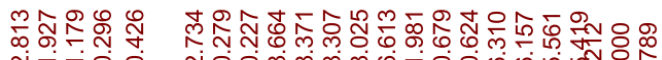

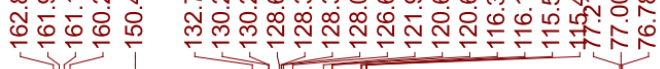
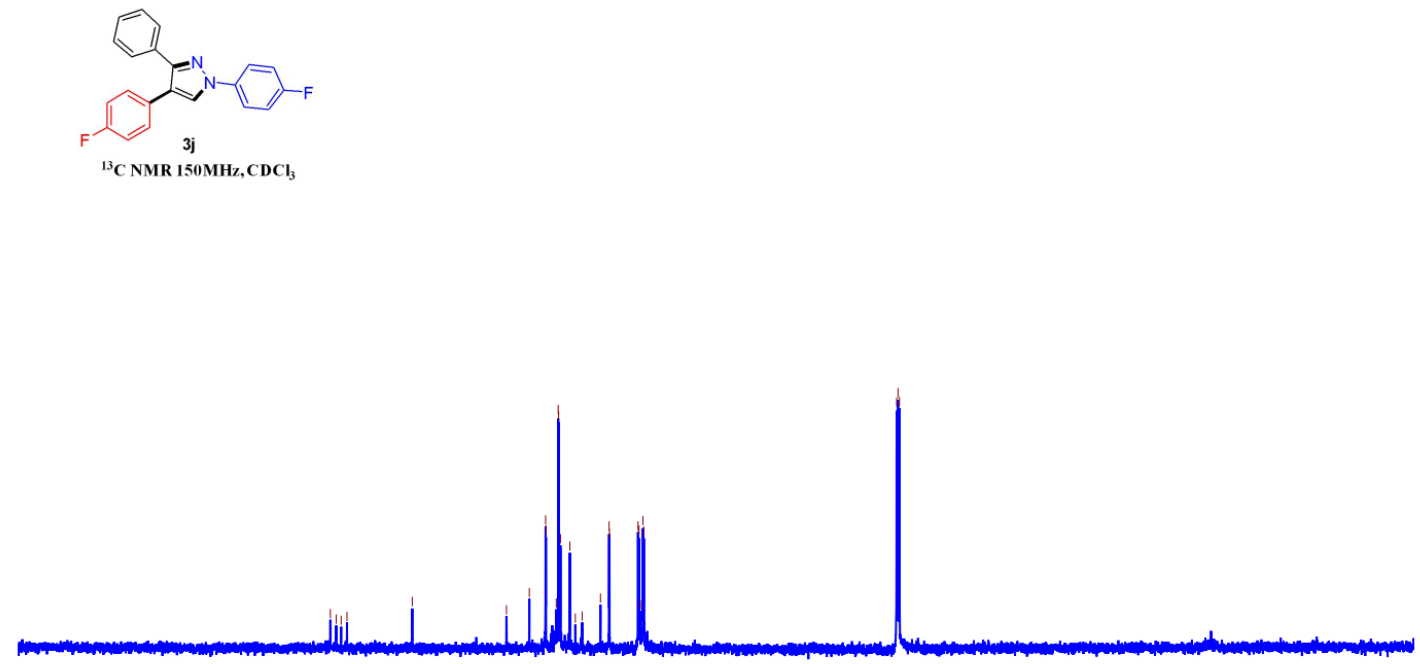

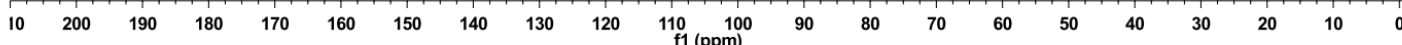



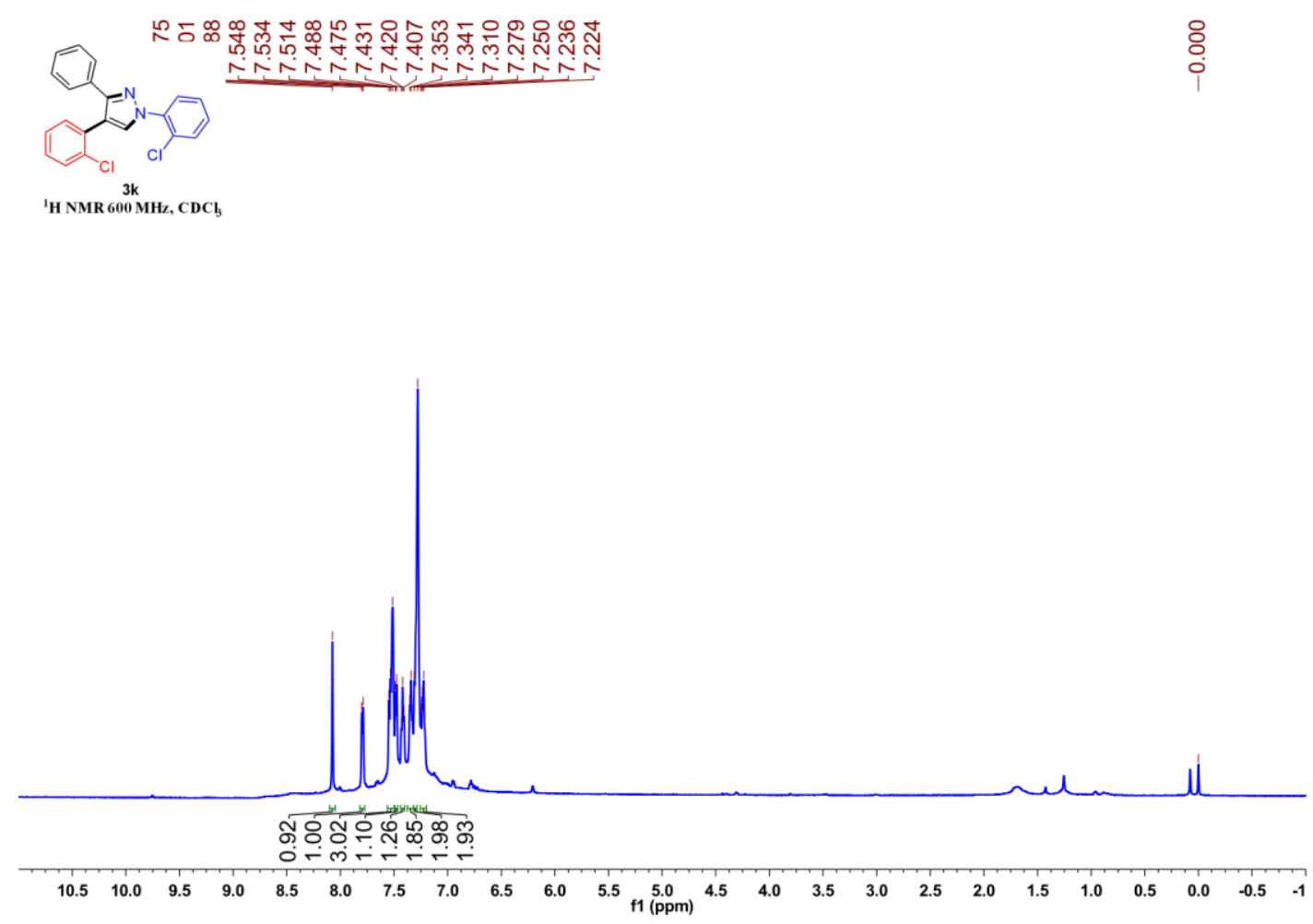

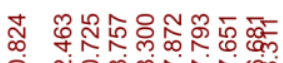

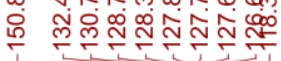

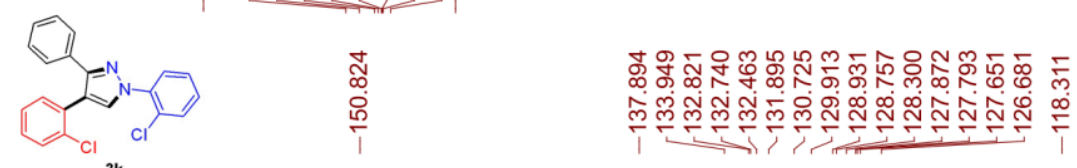

${ }^{13} \mathrm{C} \mathrm{NMR} 1500^{3 \mathrm{NH}} \mathrm{H}, \mathrm{CDCl}_{3}$

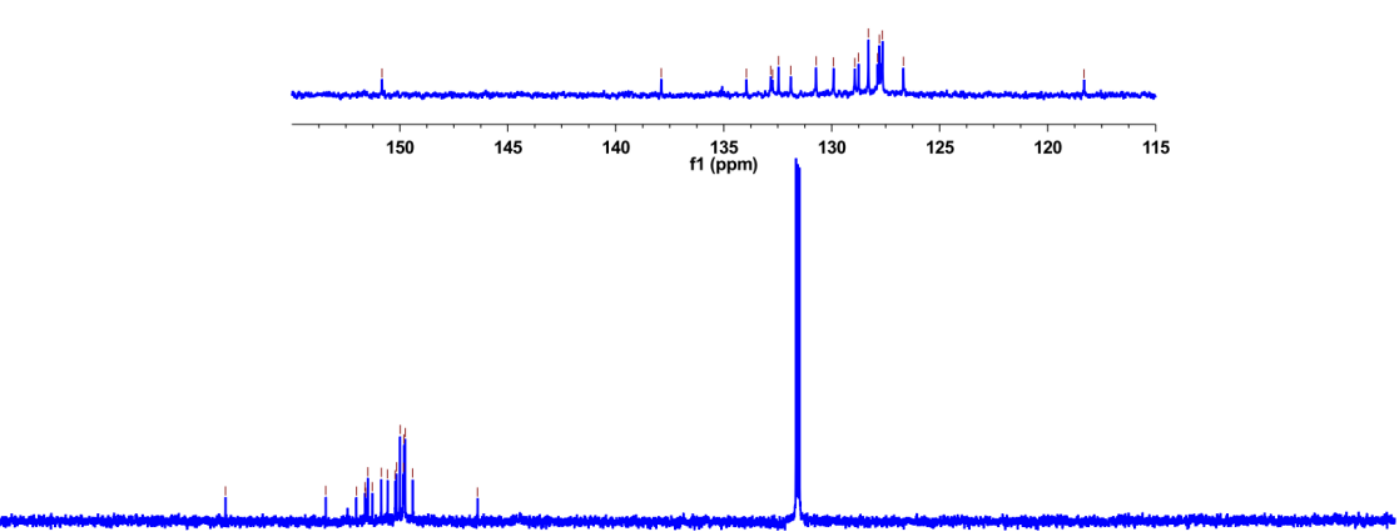

150

145

135
$\mathrm{f} 1(\mathrm{ppm})$

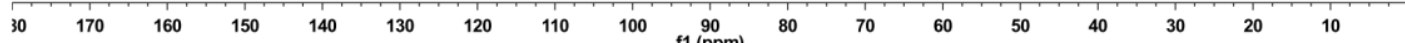




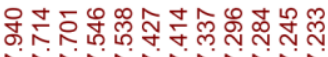
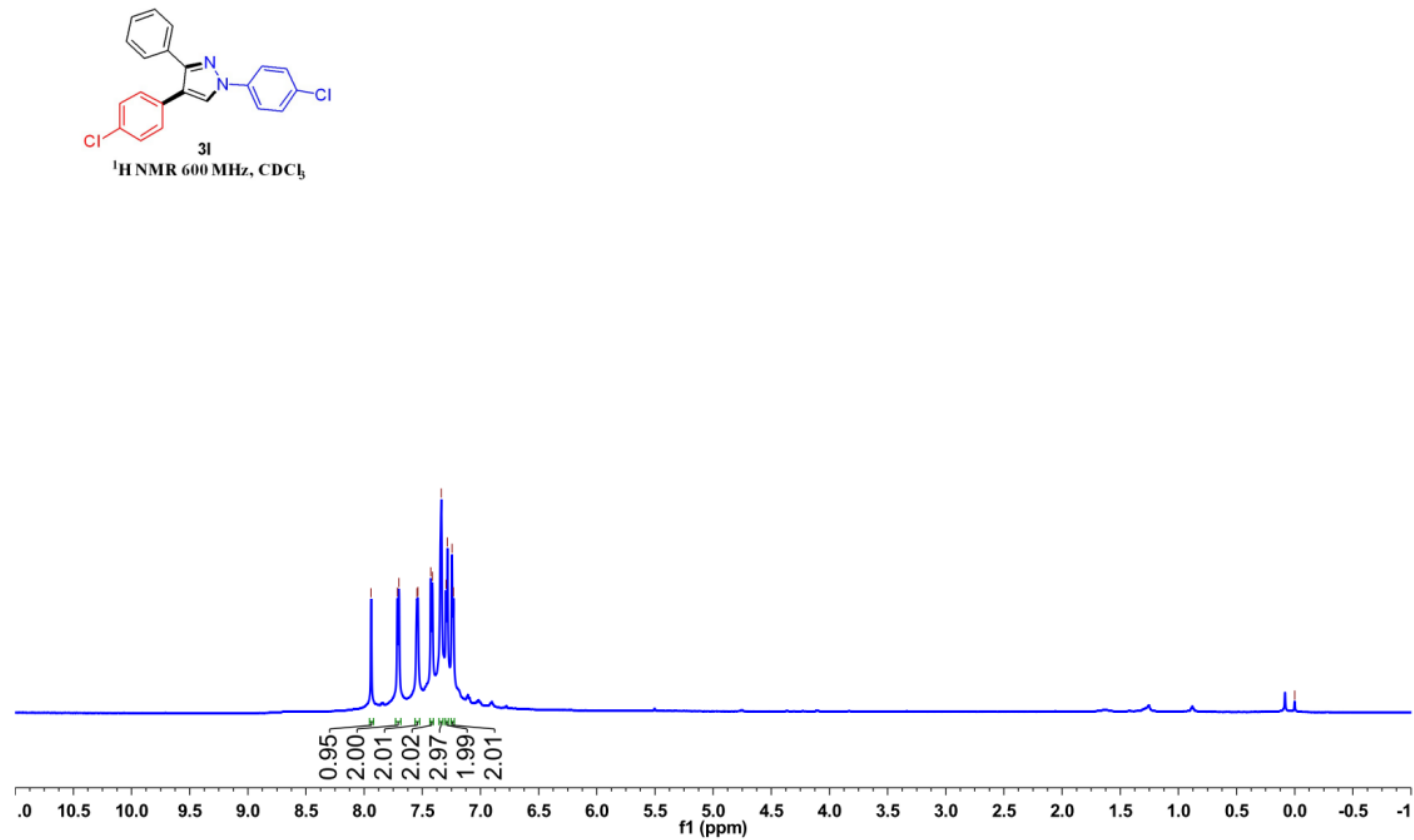

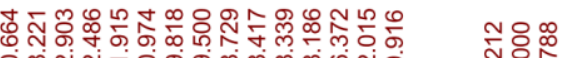

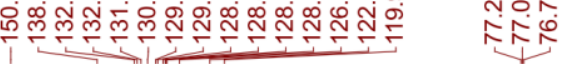

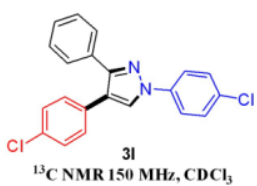

${ }^{13} \mathrm{CNMR} 150 \mathrm{MH}, \mathrm{CDCl}$

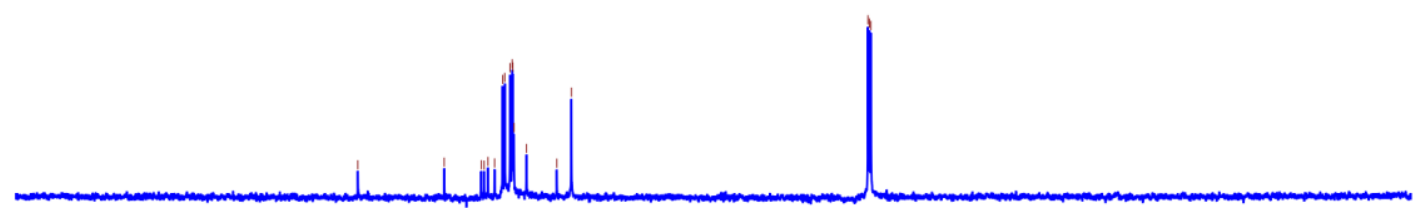

$\begin{array}{lllllllllllllllllllllllllll}10 & 190 & 180 & 170 & 160 & 150 & 140 & 130 & 120 & 110 & 100 & 90 & 80 & 70 & 60 & 50 & 40 & 30 & 20 & 10 & 0\end{array}$ 

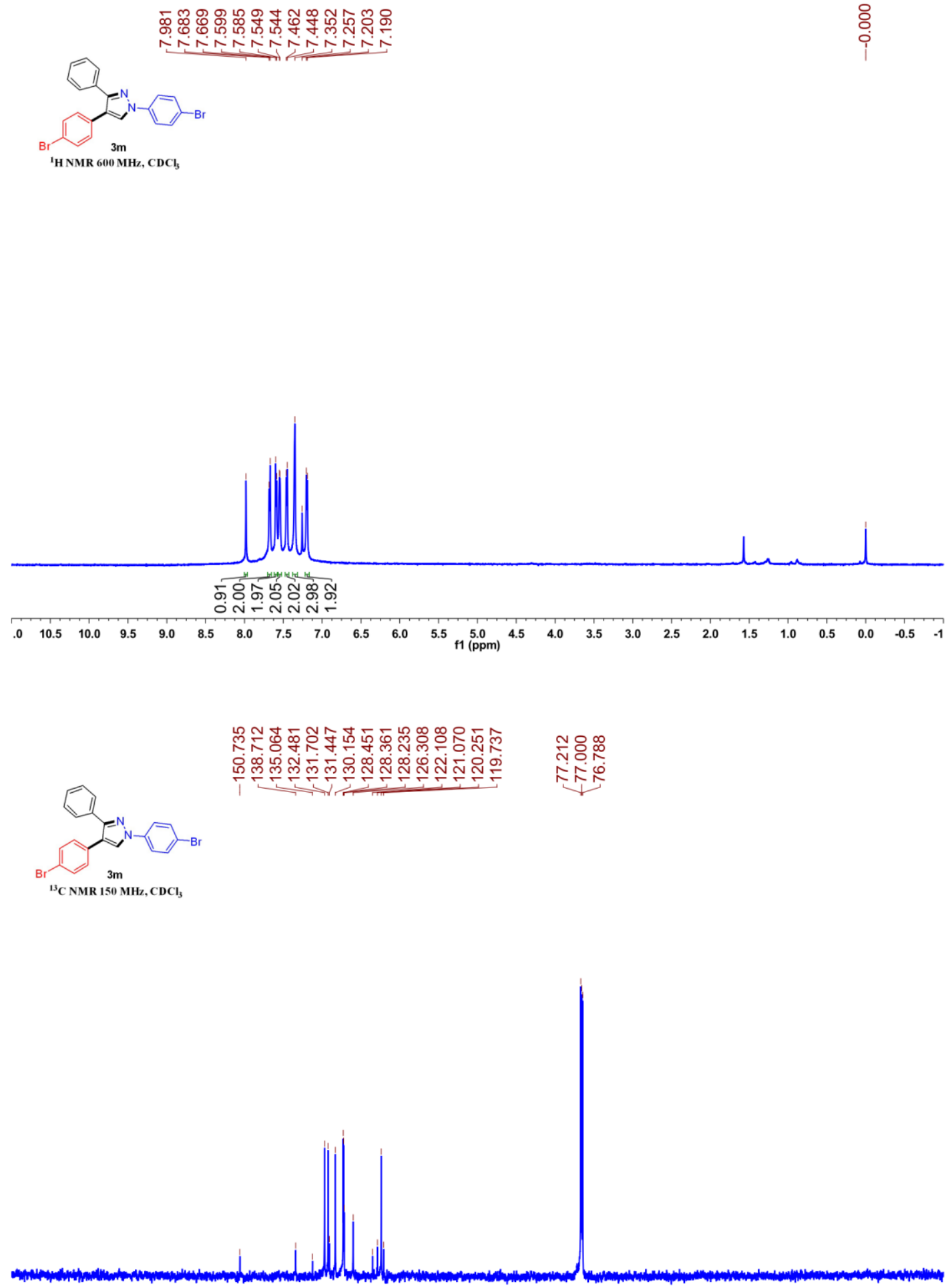

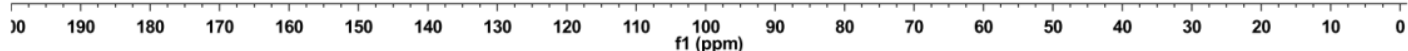




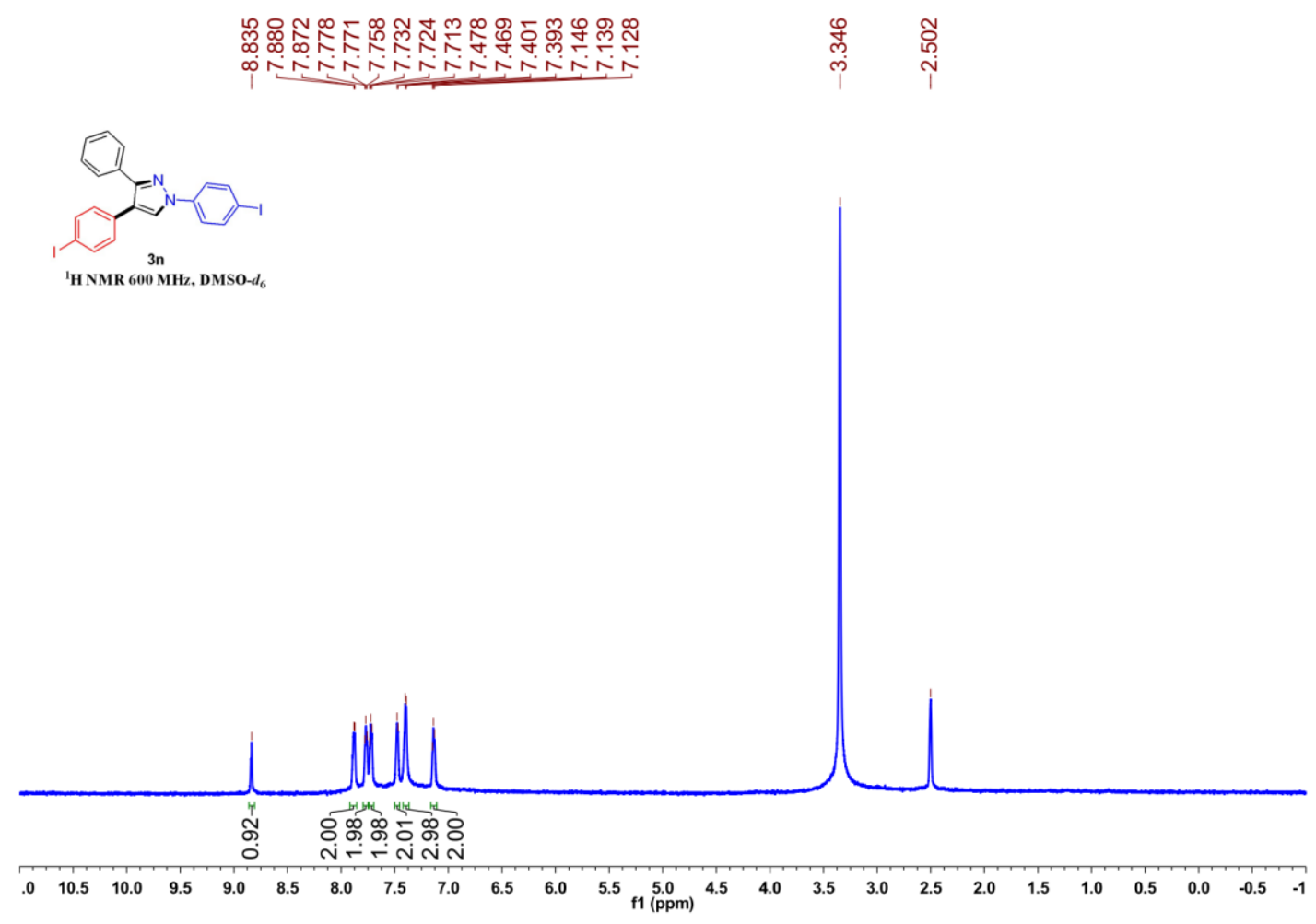

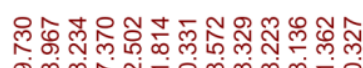

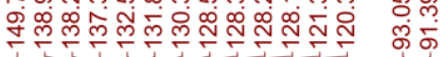

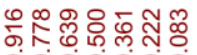

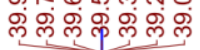

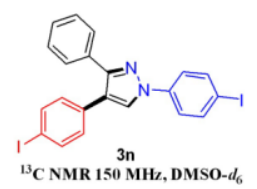

कर

${ }^{13}$ C NMR $150 \mathrm{MHz}$, DMSO- $d_{6}$

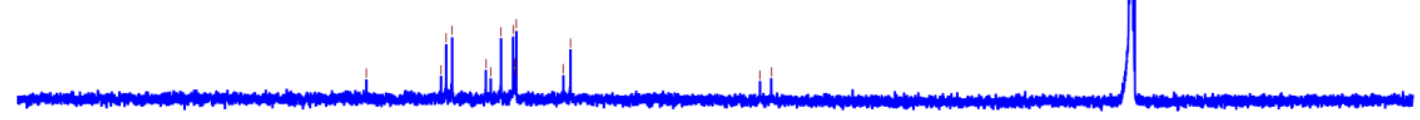

$\begin{array}{lllllllllllllllllllllllllll} & 190 & 180 & 170 & 160 & 150 & 140 & 130 & 120 & 110 & 100 & 90 & 80 & 70 & 60 & 50 & 40 & 30 & 20 & 10 & 0\end{array}$ 


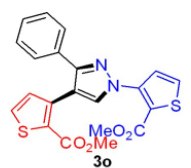

'H NMR $600 \mathrm{MHz}$, DMSO-d

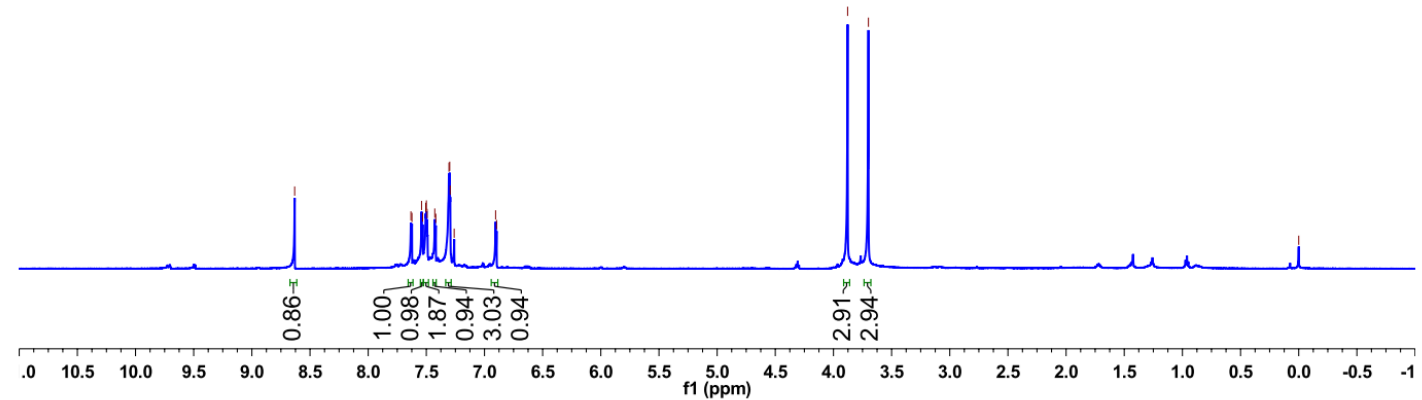

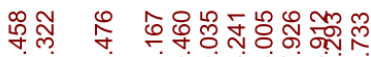

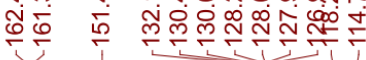

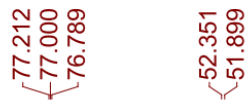

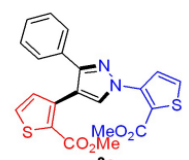

${ }^{13} \mathrm{C} \mathrm{NMR} 150 \mathrm{MHz}$, DMSO- $d_{6}$

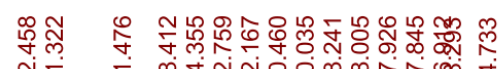

눈

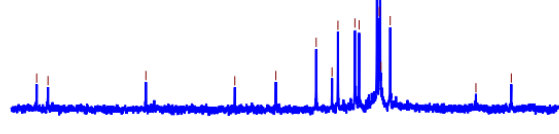

$\begin{array}{lllllllllll}165 & 160 & 155 & 150 & 145 & \begin{array}{c}140 \\ \mathrm{f} 1(\mathrm{ppm})\end{array} & 130 & 125 & 120 & 115 & 110\end{array}$

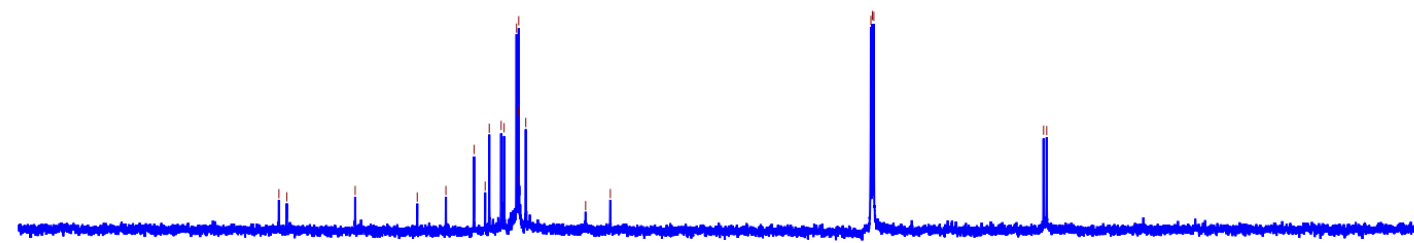

$\begin{array}{lllllllllllllllllllllll}30 & 190 & 180 & 170 & 160 & 150 & 140 & 130 & 120 & 110 & 100 & 90 & 80 & 70 & 60 & 50 & 40 & 30 & 20 & 10 & 0\end{array}$ 


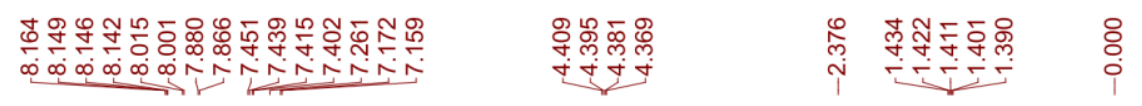
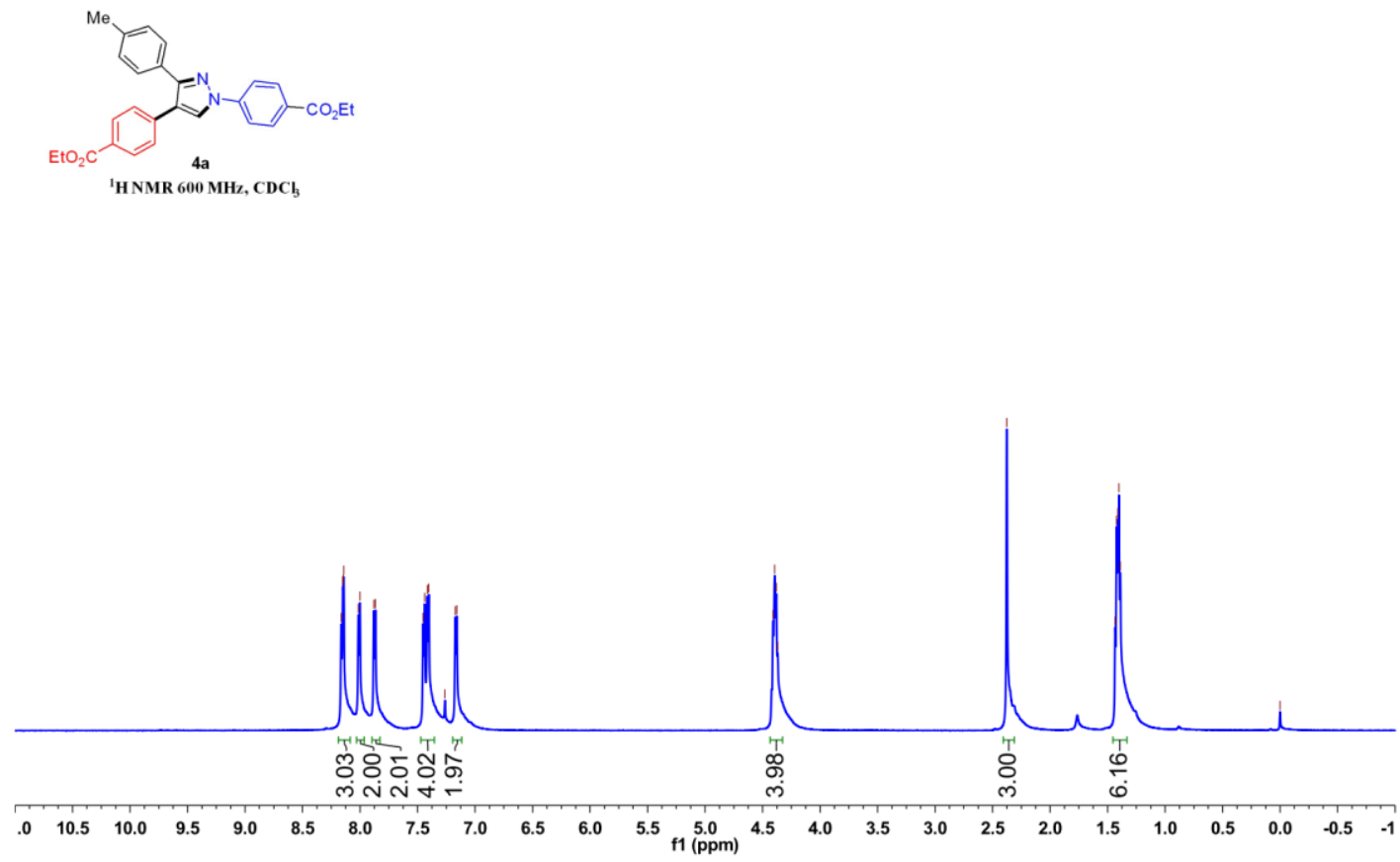

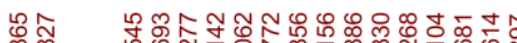

ำㅇำ

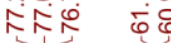

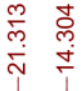

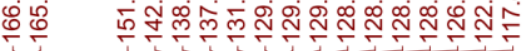
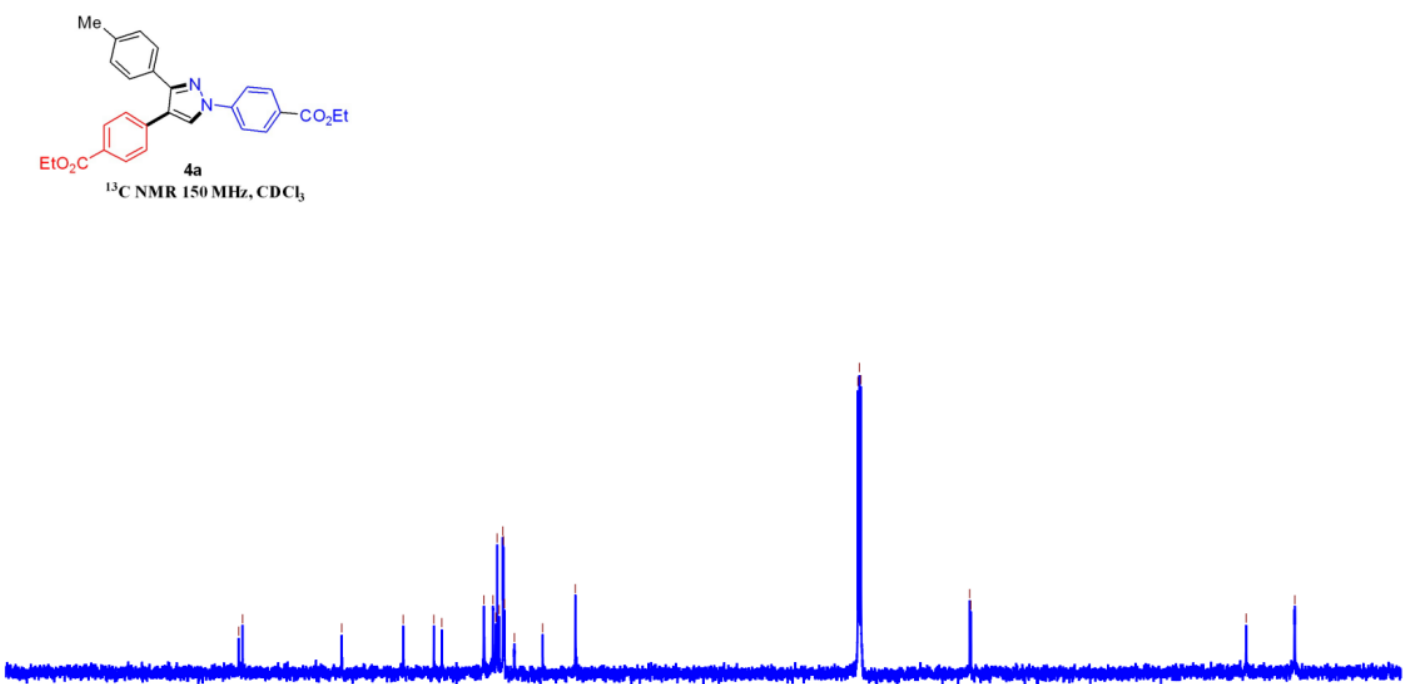

$\begin{array}{llllllllllllllllllllllllllllll} & 190 & 180 & 170 & 160 & 150 & 140 & 130 & 120 & 110 & 100 & 90 & 80 & 70 & 60 & 50 & 40 & 30 & 20 & 10 & 0\end{array}$ 


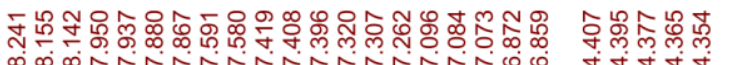

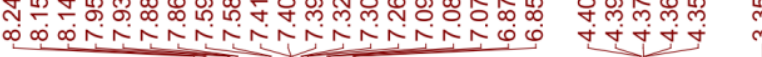

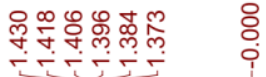<smiles>CCOC(=O)C=C(c1ccc(OCC)cc1)c1ccccc1OC</smiles>

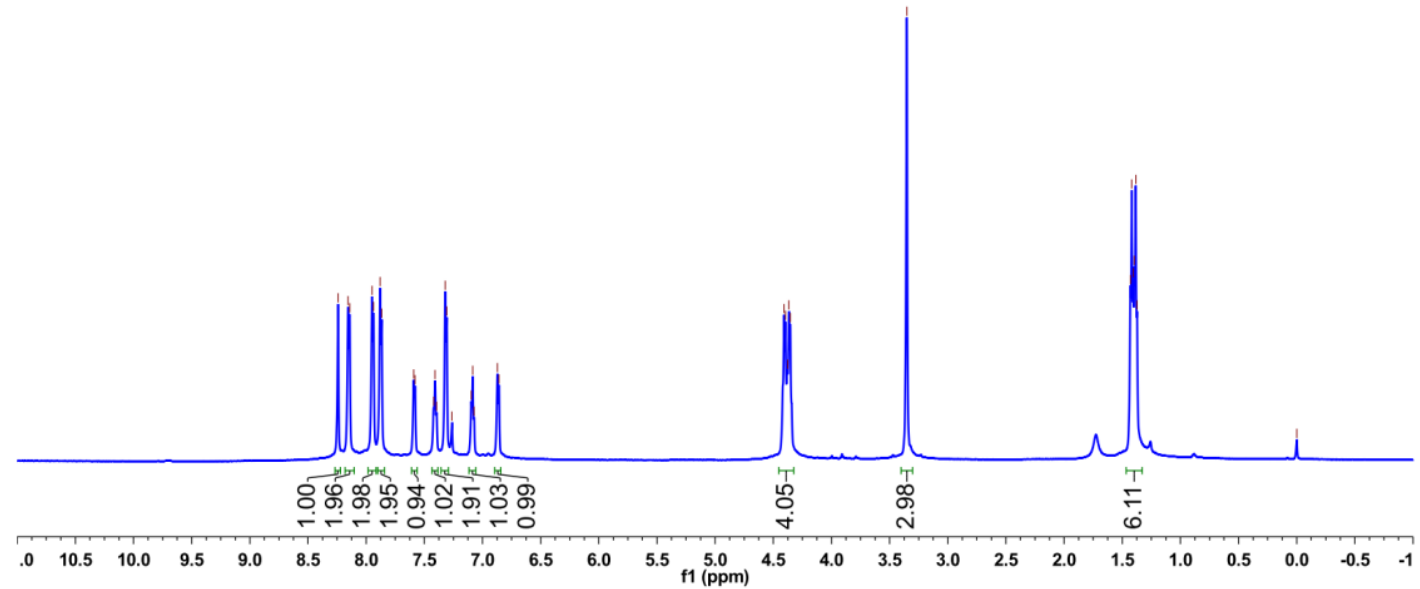

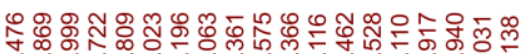

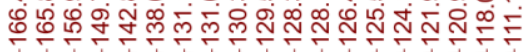

궁ำ

神 它它

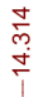
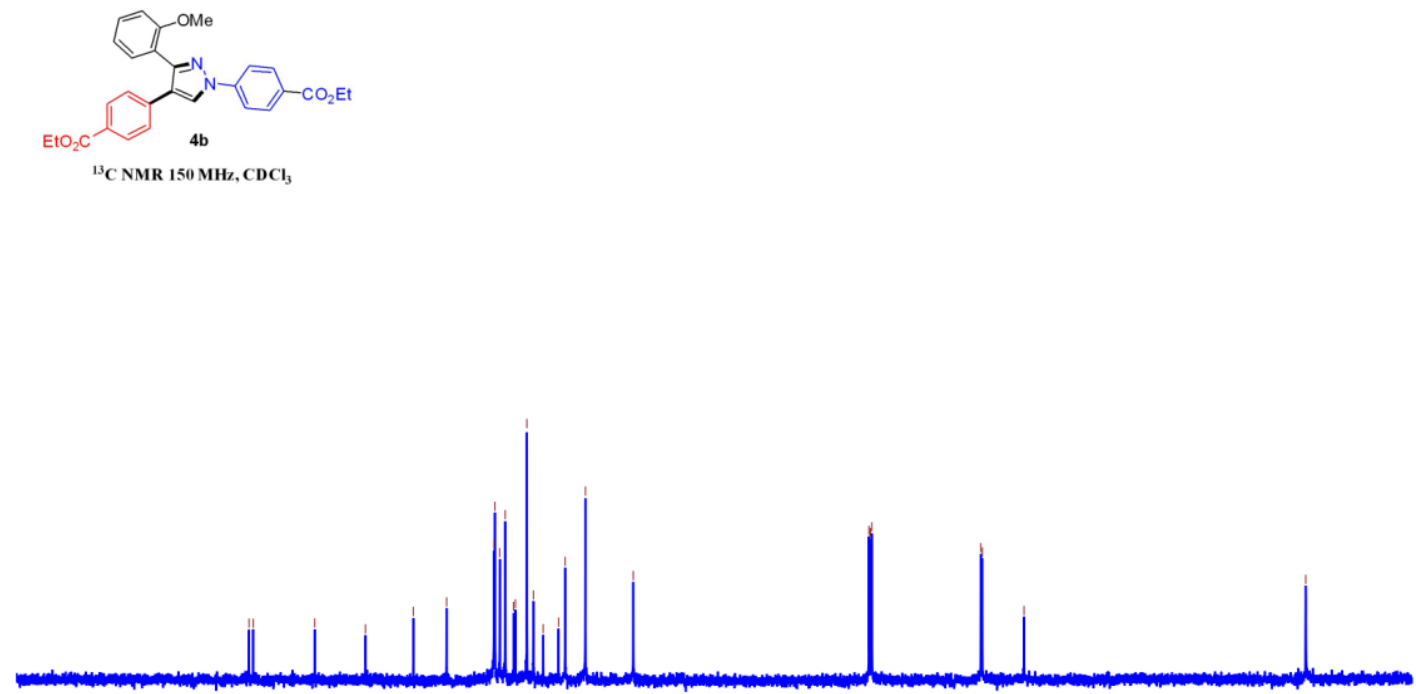

\begin{tabular}{llllllllllllllllllllllll}
\hline 0 & 190 & 180 & 170 & 160 & 150 & 140 & 130 & 120 & 110 & 100 & 90 & 80 & 70 & 60 & 50 & 40 & 30 & 20 & 10 & 0
\end{tabular} 

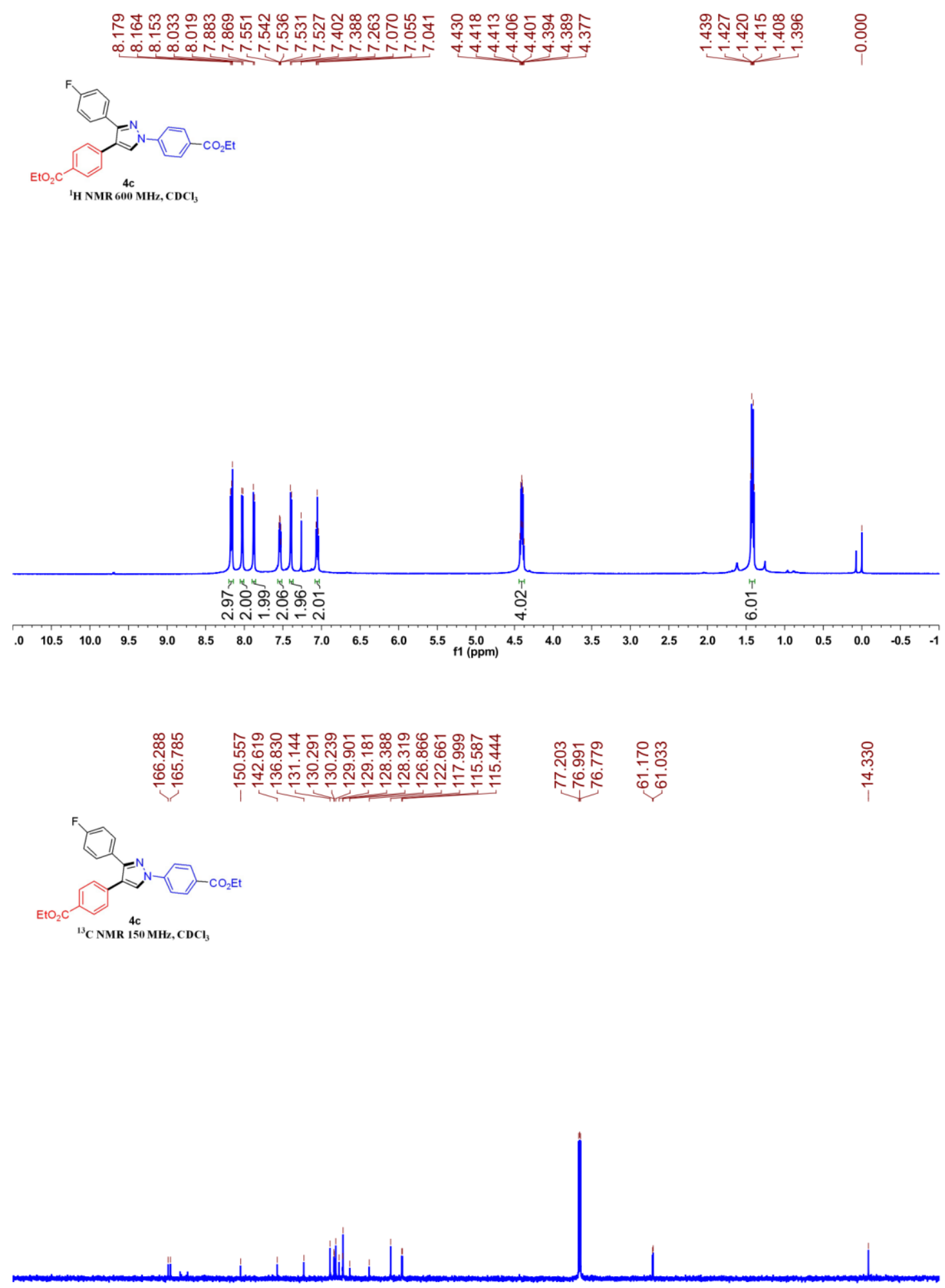

$\begin{array}{llllllllllllllllllllll} & 190 & 180 & 170 & 160 & 150 & 140 & 130 & 120 & 110 & 100 & 100 & 80 & 70 & 60 & 50 & 40 & 30 & 20 & 10 & 0\end{array}$ 

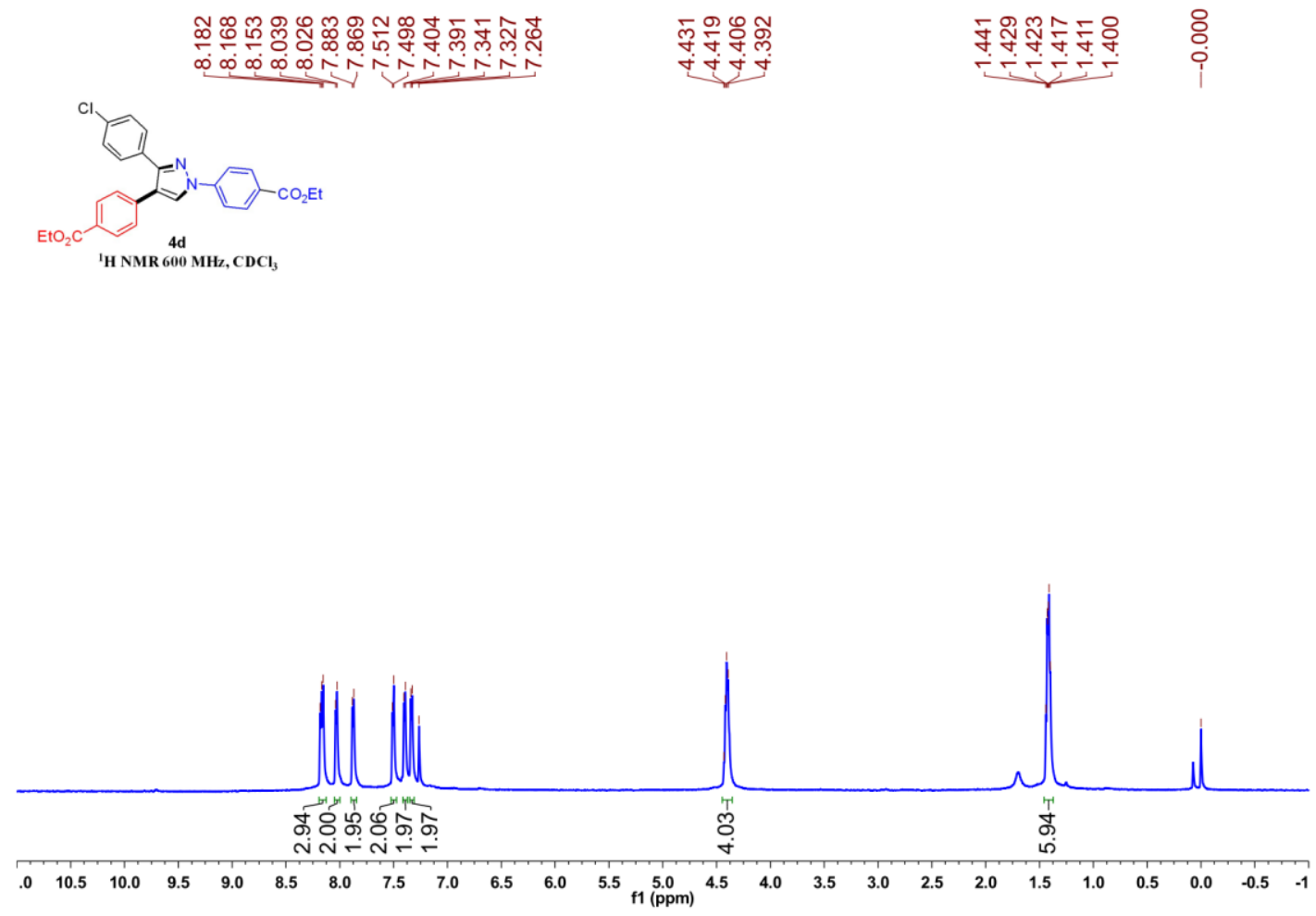

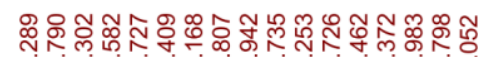

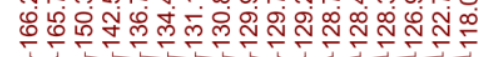

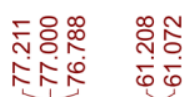

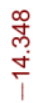<smiles></smiles>

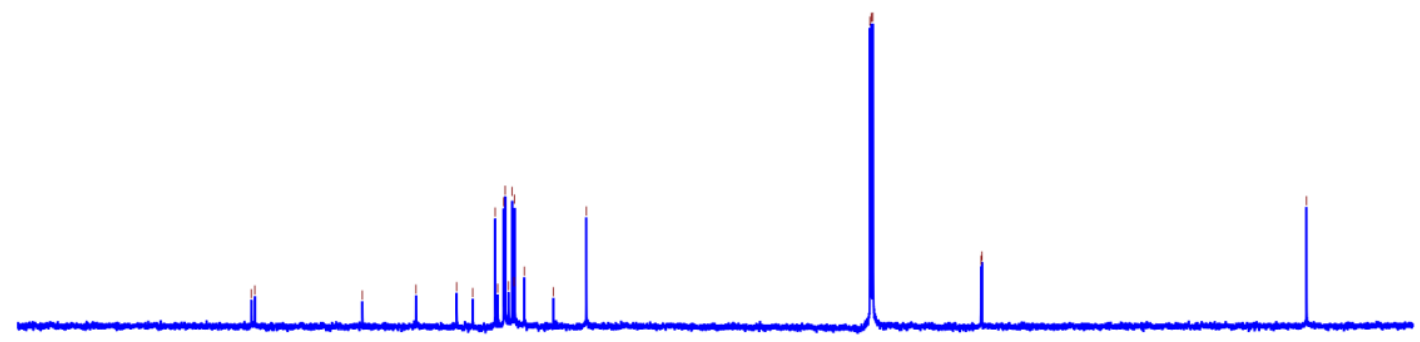

$\begin{array}{llllllllllllllllllllll} & 190 & 180 & 170 & 160 & 150 & 140 & 130 & 120 & 110 & 100 & 100 & 80 & 70 & 60 & 50 & 40 & 30 & 20 & 10 & 0\end{array}$ 

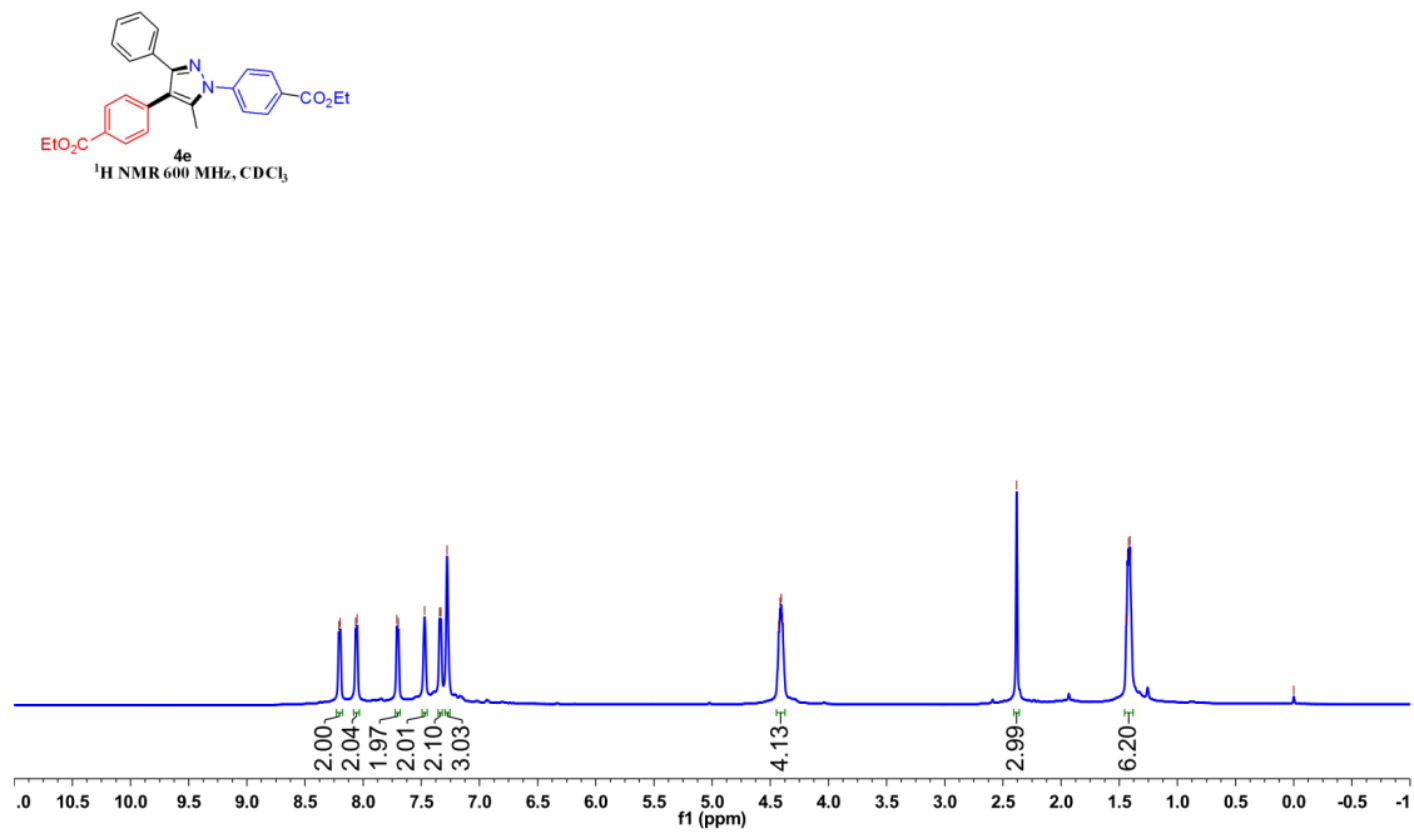

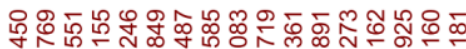

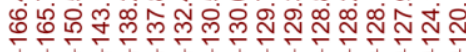

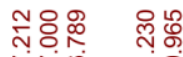

NN⿱中⿰㇀丶㇀

్ํ욤

ㅍำ

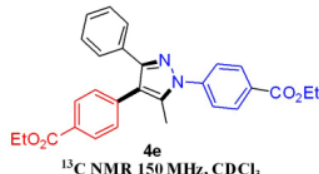

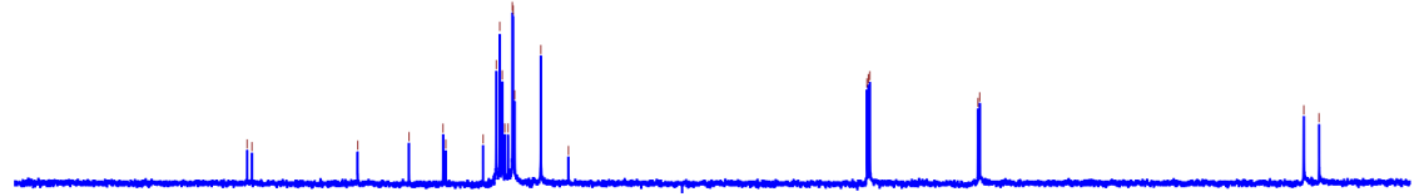

$\begin{array}{lllllllllllllllllllllll}30 & 190 & 180 & 170 & 160 & 150 & 140 & 130 & 120 & 110 & \begin{array}{l}100 \\ \mathrm{f} 1(\mathrm{ppm})\end{array} & 90 & 80 & 70 & 60 & 50 & 40 & 30 & 20 & 10 & 0\end{array}$ 


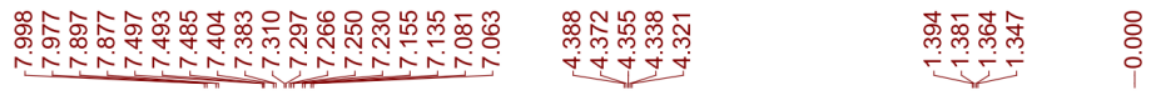<smiles>CCOC(=O)c1ccc(N2C(c3ccccc3)=C(c3ccc(OCC)cc3)N(c3ccccc3)C2c2ccccc2)cc1</smiles>

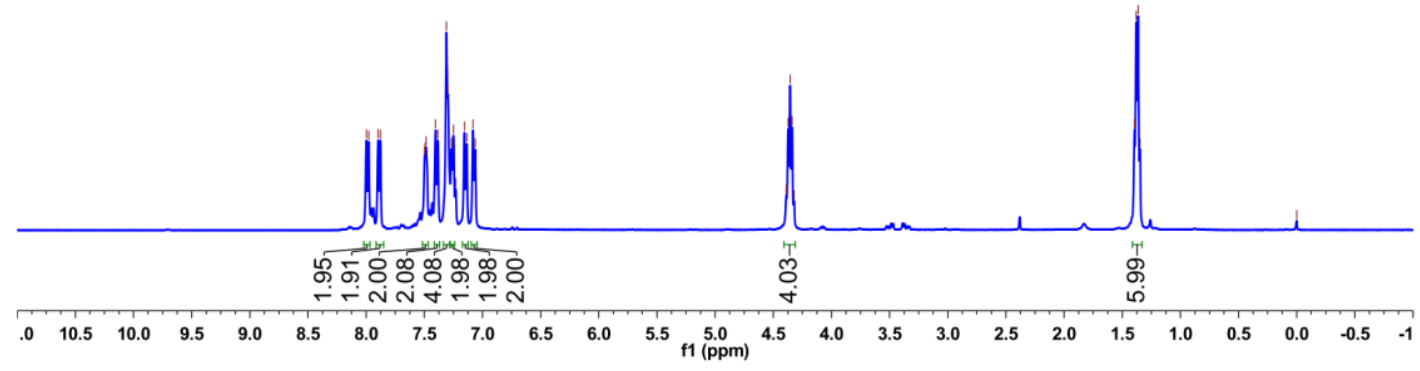

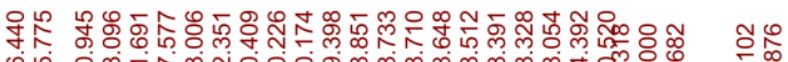

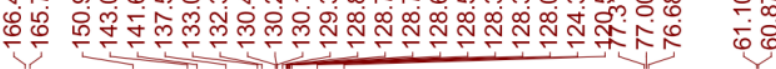

ণ্ণ<smiles>CCOC(=O)c1ccc(-c2c(-c3ccccc3)nn(-c3ccc(C(=O)O)cc3)c2-c2ccccc2)cc1</smiles>

${ }^{3} \mathrm{C} \mathrm{NMR} 100 \mathrm{MHz}_{2} \mathrm{CDCl}_{3}$

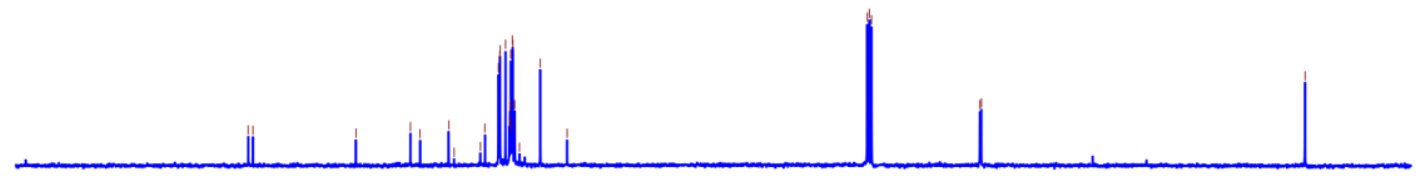

$\begin{array}{llllllllllllllllllllllllllll} & 190 & 180 & 170 & 160 & 150 & 140 & 130 & 120 & 110 & 100 & 90 & 80 & 70 & 60 & 50 & 40 & 30 & 20 & 10 & 0\end{array}$ 


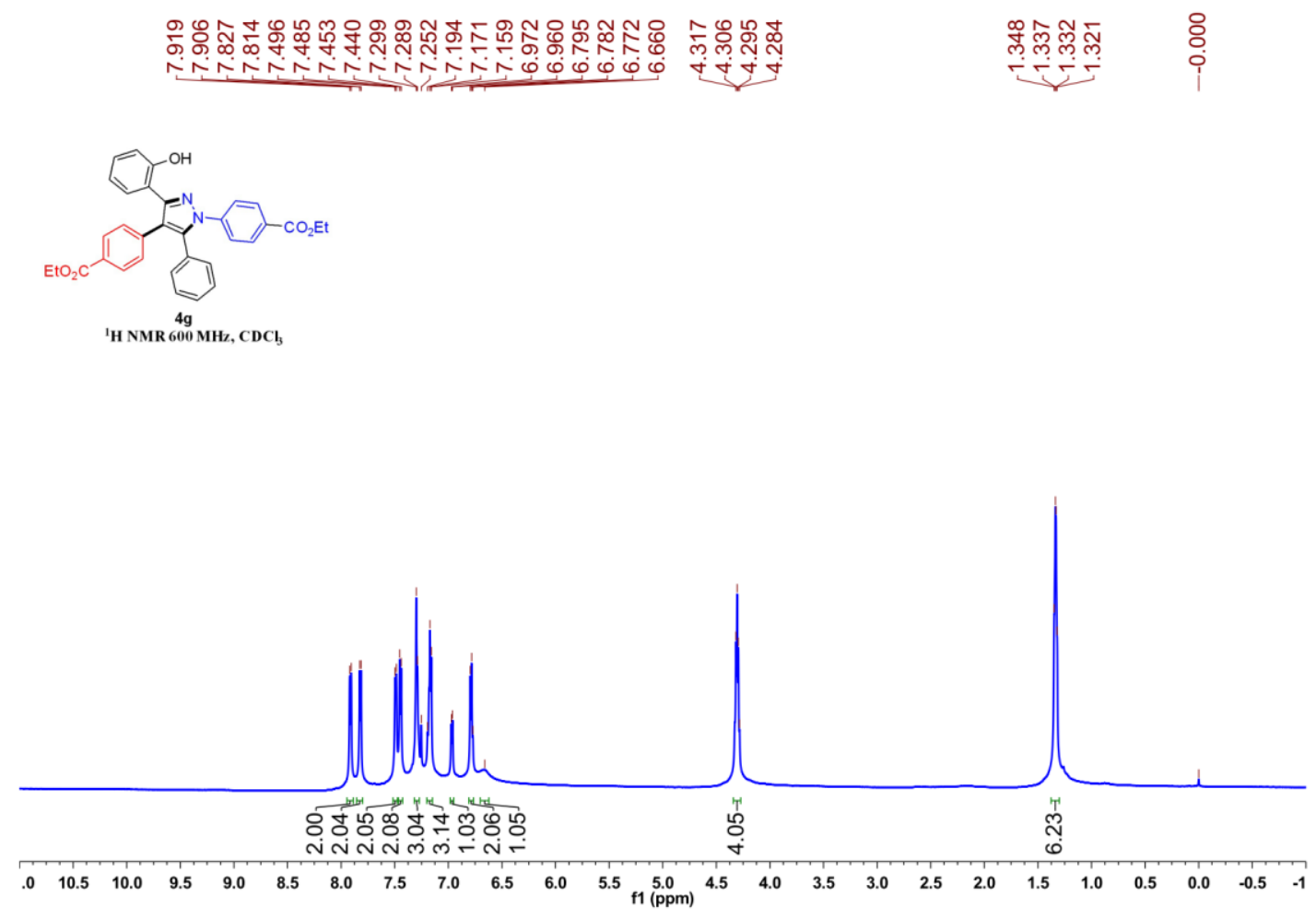

응 응

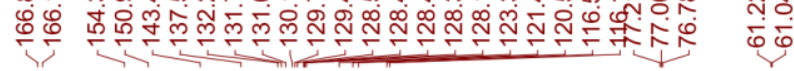

ํํํ

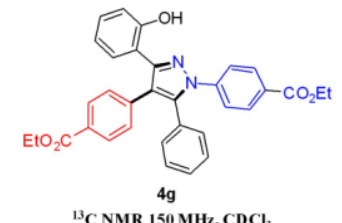

${ }^{13} \mathrm{C}$ NMR $150 \mathrm{MHz}, \mathrm{CDCl}$,

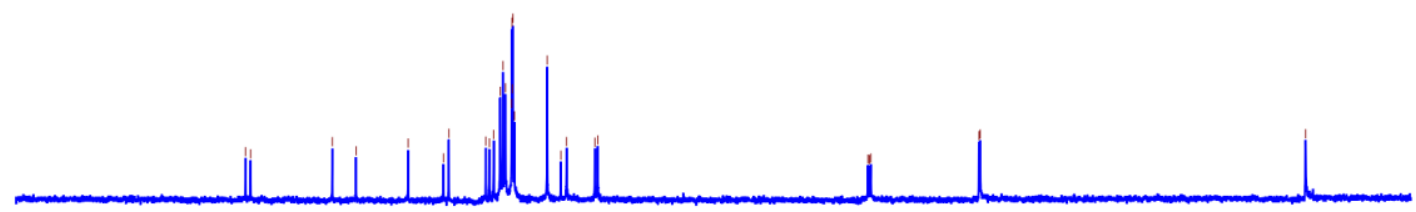

$\begin{array}{lllllllllllllllllllllllllll} & 190 & 180 & 170 & 160 & 150 & 140 & 130 & 120 & 110 & 100 & 100 & 80 & 70 & 60 & 50 & 40 & 30 & 20 & 10 & 0\end{array}$ 


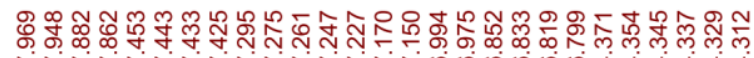
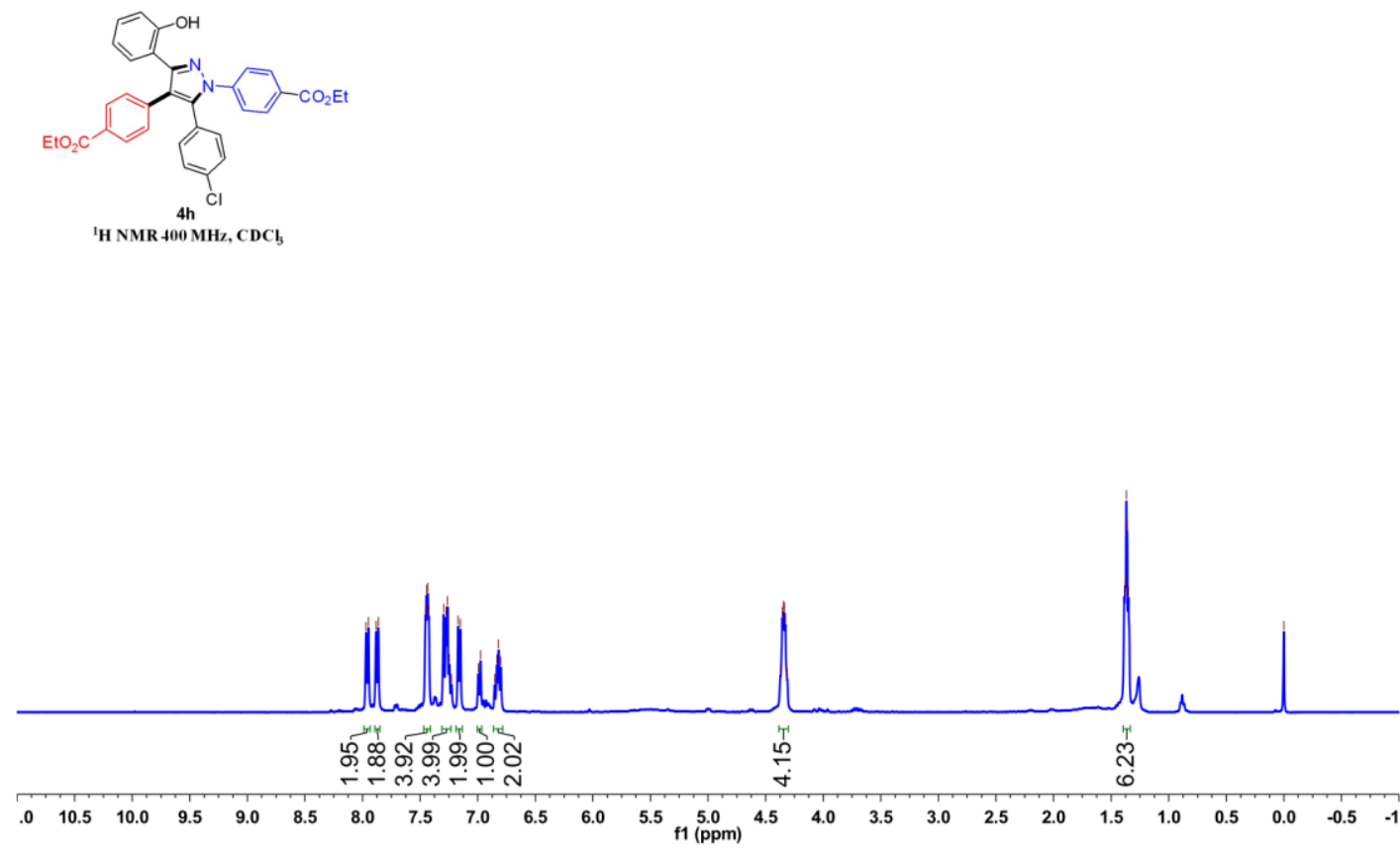

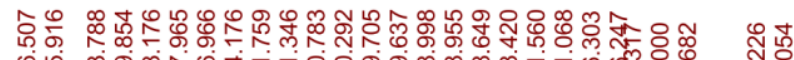

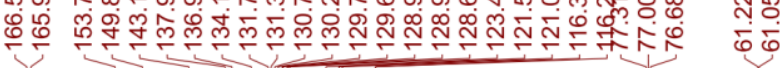

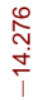

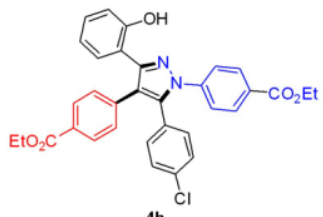

${ }^{13} \mathrm{CNMR}_{100 \mathrm{MHz}} \mathrm{CDCl}_{3}$

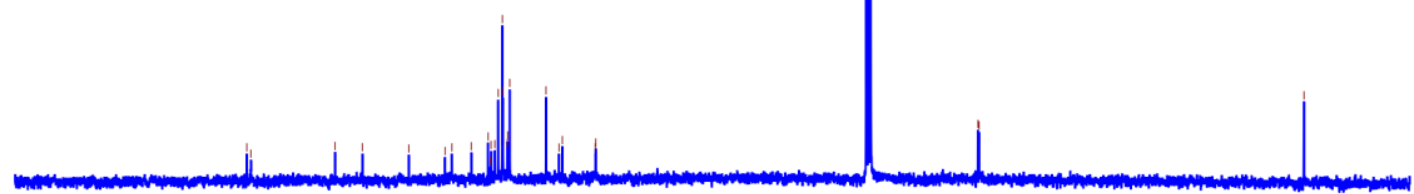

$\begin{array}{lllllllllllllllllllllllllllll}10 & 190 & 180 & 170 & 160 & 150 & 140 & 130 & 120 & 110 & 100 & 90 & 80 & 70 & 60 & 50 & 40 & 30 & 20 & 10 & 0\end{array}$ 

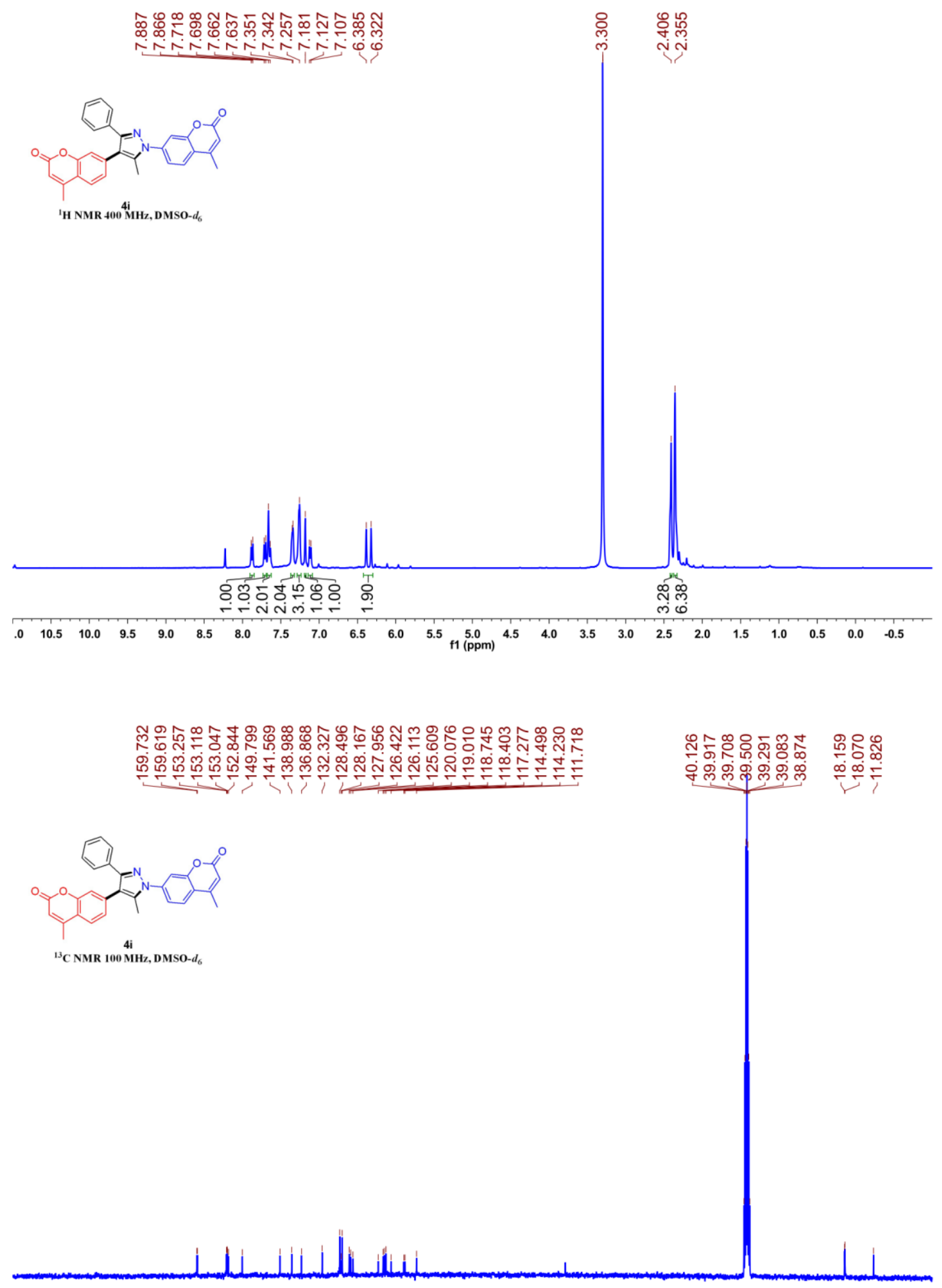

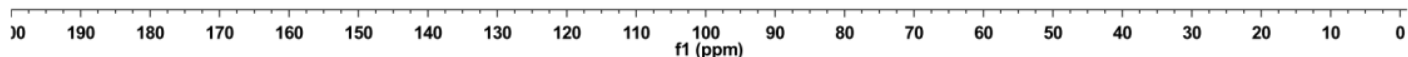




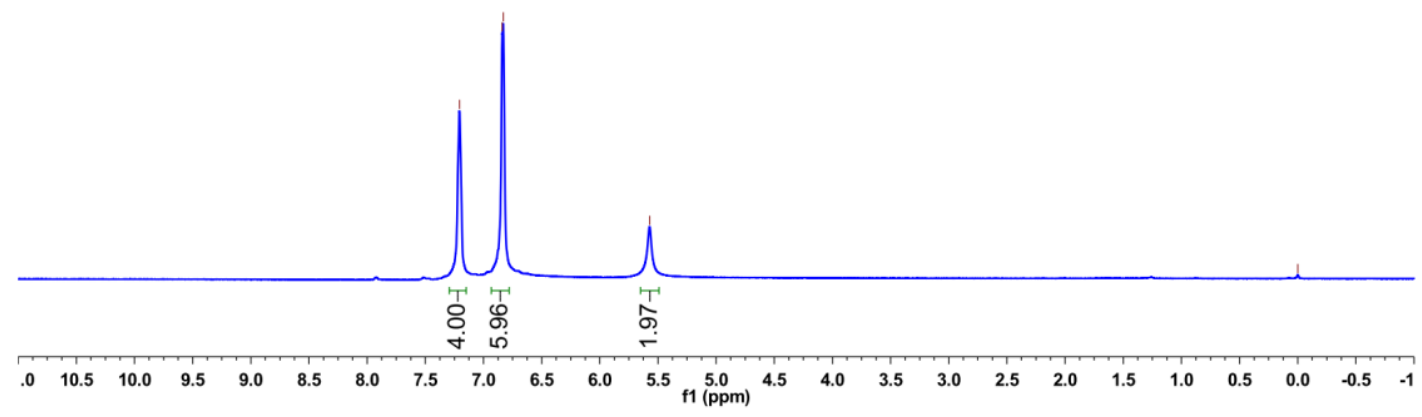

$\mathrm{C}^{\mathrm{N}}$

${ }^{13} \mathrm{C}$ NMR $150 \mathrm{MHz}, \mathrm{CDCl}$

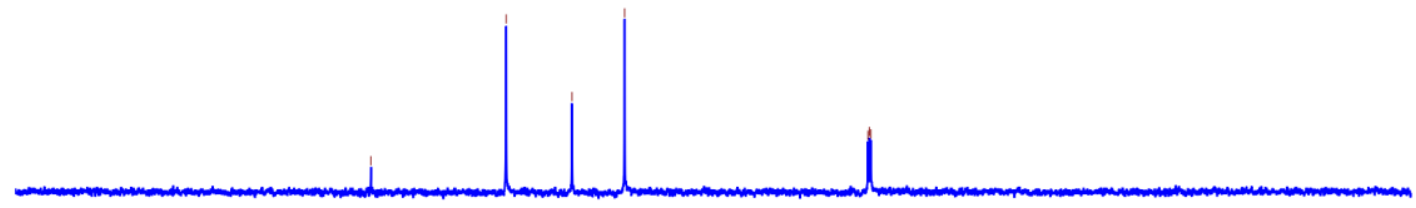

$\begin{array}{llllllllllllllllllllllllllllll} & 190 & 180 & 170 & 160 & 150 & 140 & 130 & 120 & 110 & 100 & 90 & 80 & 70 & 60 & 50 & 40 & 30 & 20 & 10 & 0\end{array}$ 